\title{
Total Synthesis of Stephanotic Acid Methyl Ester
}

David J. Bentley, Alexandra M. Z. Slawin, and Christopher J. Moody

\section{SUPPORTING INFORMATION}

\section{TABLE OF CONTENTS}

General experimental details

Experimental protocols and characterization data

X-ray crystal data of $( \pm)-12$;

References
S2

S2-S26

S26-S29

S29 


\section{General Experimental Details}

Commercially available reagents were used throughout without purification unless otherwise stated. Light petroleum refers to the fraction with bp $40-60{ }^{\circ} \mathrm{C}$ and was distilled before use. Ether refers to diethyl ether. Reactions were routinely carried out under a nitrogen or argon atmosphere. Analytical thin layer chromatography was carried out on aluminum backed plates coated with Merck Kieselgel $60 \mathrm{GF}_{254}$, and visualized under UV light at 254 and/or $360 \mathrm{~nm}$. Flash chromatography was carried out using Merck Kieselgel $60 \mathrm{H}$ silica or Matrex silica 60. Fully characterized compounds were chromatographically homogeneous.

Infrared spectra were recorded in the range $4000-600 \mathrm{~cm}^{-1}$ using a Nicolet Magna FT-550 or Perkin Elmer FT-1600 spectrometers. NMR spectra were carried out on Bruker 300 and 400 $\mathrm{MHz}$ instruments $\left({ }^{1} \mathrm{H}\right.$ frequencies, corresponding ${ }^{13} \mathrm{C}$ frequencies are 75 and $100 \mathrm{MHz}$ ).

Chemical shifts are quoted in ppm with TMS as internal standard. $J$ values are recorded in Hz. In the ${ }^{13} \mathrm{C}$ spectra, signals corresponding to $\mathrm{CH}, \mathrm{CH}_{2}$ or $\mathrm{CH}_{3}$ groups, as assigned from DEPT, are noted; all others are C. High and low resolution mass spectra were recorded on a Micromass GCT TDF High Resolution mass spectrometer, or at the EPSRC Mass Spectrometry Service (Swansea). Specific rotations were measured on an AA-1000 polarimeter and values are quoted in $10^{-1} \mathrm{degcm}^{2} \mathrm{~g}^{-1}$.

\section{Indole-6-carbonitrile}<smiles>N#Cc1ccc2cc[nH]c2c1</smiles>

Dimethylformamide dimethylacetal $(32.0 \mathrm{ml}, 241 \mathrm{mmol})$ was added to 4-methyl-3-nitrobenzonitrile $(15.0 \mathrm{~g}, 92.6 \mathrm{mmol})$ in DMF $(450 \mathrm{ml})$ and the mixture was heated 
under reflux for $15 \mathrm{~h}$. The reaction mixture was concentrated and the crude enamine split into two equal batches. Each batch was dissolved in anhydrous THF $(150 \mathrm{ml})$ and added to a Parr tube containing palladium on carbon $(10 \% ; 7.50 \mathrm{~g})$. The solutions were degassed and stirred under hydrogen pressure (50 psi) for $30 \mathrm{~min}$. The reaction mixtures were filtered through Celite and the filtrates were combined and concentrated. The residue was dissolved in ethyl acetate $(500 \mathrm{ml})$, washed with brine $(2 \square 300 \mathrm{ml})$, dried $\left(\mathrm{MgSO}_{4}\right)$ and concentrated. The residue was purified by column chromatography (dichloromethane) to give the title compound ( $9.07 \mathrm{~g}, 69 \%$ ) as a yellow powder; mp $126-127^{\circ} \mathrm{C}$ (from ethyl acetate / light petroleum), (lit., ${ }^{1} \mathrm{mp} 132{ }^{\circ} \mathrm{C}$ ); (Found: $\mathrm{MH}^{+}$, 143.0612. $\mathrm{C}_{9} \mathrm{H}_{6} \mathrm{~N}_{2}+\mathrm{H}$ requires 143.0609); $\square_{\max }(\mathrm{KBr}) / \mathrm{cm}^{-1} 3412(\mathrm{NH}), 2219(\mathrm{C} \equiv \mathrm{N}) ; \square_{\mathrm{H}}(300$ $\left.\mathrm{MHz} ; \mathrm{CDCl}_{3}\right) 8.76(1 \mathrm{H}, \mathrm{s}, \mathrm{NH}), 7.78(1 \mathrm{H}, \mathrm{s}, 7-\mathrm{H}), 7.69(1 \mathrm{H}, \mathrm{d}, J$ 8.2, 4-H), $7.43(1 \mathrm{H}$, app t, $J$ 2.7, 2-H), 7.34 (1H, dd, J 8.2, 0.9, 5-H), 6.63 (1H, m, 3-H); [c (75 MHz; $\left.\mathrm{CDCl}_{3}\right) 133.9$ (C), 130.5 (C), $127.5(\mathrm{CH}), 122.0(\mathrm{CH}), 120.9(\mathrm{CH}), 120.2(\mathrm{C}), 115.4(\mathrm{CH}), 103.4(\mathrm{C}), 102.7(\mathrm{CH}) ; m / z(\mathrm{CI})$ $143\left(\mathrm{MH}^{+}, 100 \%\right), 142\left(\mathrm{M}^{+}, 28\right)$.

\section{N-tert-Butoxycarbonyl-6-isobutyrylindole 7}<smiles>CC(C)C(=O)c1ccc2cc[nH]c2c1</smiles><smiles>CC(C)C(=O)c1ccc2ccn(C(C)(C)C)c2c1</smiles>

(a) Isopropylmagnesium chloride in THF $(2.0 \mathrm{M} ; 400 \mathrm{ml}, 800 \mathrm{mmol})$ was added to a solution of indole-6-carbonitrile $(9.07 \mathrm{~g}, 63.9 \mathrm{mmol})$ in anhydrous THF $(50 \mathrm{ml})$ at $0{ }^{\circ} \mathrm{C}$. The reaction mixture was heated under reflux for $24 \mathrm{~h}$. The reaction mixture was cooled to $0{ }^{\circ} \mathrm{C}$, hydrochloric acid ( $2 \mathrm{M} ; 400 \mathrm{ml}$ ) was added slowly until $\mathrm{pH} 4$ was reached, and then the mixture was allowed to stir at room temperature for $30 \mathrm{~min}$. The THF was removed under vacuum and the product was 
extracted from the aqueous with ether $(2 \square 500 \mathrm{ml})$. The combined organics were washed with brine $(500 \mathrm{ml})$, dried $\left(\mathrm{MgSO}_{4}\right)$ and concentrated.

The procedure above was repeated using a second batch of 6-cyanoindole $(8.52 \mathrm{~g}, 60.0 \mathrm{mmol})$, and the two crude products were combined and purified by recrystallization from dichloromethane. This recrystallized product was combined with a further quantity obtained by column chromatography (dichloromethane) of the mother liquor to give 6-isobutyrylindole (15.7 $\mathrm{g}, 68 \%$ ) as a colorless powder; $\mathrm{mp} 165-167^{\circ} \mathrm{C}$ (from ethyl acetate / light petroleum); (Found: $\mathrm{C}$, 77.1; H, 7.0; N, 7.4. $\mathrm{C}_{12} \mathrm{H}_{13} \mathrm{NO}$ requires C, 77.0; H, 7.0; N, 7.5\%); (Found: $\mathrm{MH}^{+}, 188.1079$. $\mathrm{C}_{12} \mathrm{H}_{13} \mathrm{NO}+\mathrm{H}$ requires 188.1075); $\square_{\max }(\mathrm{KBr}) / \mathrm{cm}^{-1} 3312(\mathrm{NH}), 2981(\mathrm{CH}), 1647(\mathrm{CO})$; $\square_{\mathrm{H}}(300$ $\left.\mathrm{MHz} ; \mathrm{CDCl}_{3}\right) 8.73(1 \mathrm{H}, \mathrm{br} \mathrm{s}, \mathrm{NH}), 8.14(1 \mathrm{H}, \mathrm{s}, 7-\mathrm{H}), 7.77(1 \mathrm{H}, \mathrm{dd}, J$ 8.4, 1.3, 4-H), $7.68(1 \mathrm{H}, \mathrm{d}$, $J$ 8.4, 5-H), $7.40\left(1 \mathrm{H}\right.$, app t, $J$ 2.8, 2-H), $6.60(1 \mathrm{H}, \mathrm{m}, 3-\mathrm{H}), 3.66\left(1 \mathrm{H}, \mathrm{m}, J 6.8, \mathrm{C}_{\mathrm{HMe}}\right), 1.25$ (6H, d, J 6.8 CHMe 2 ); Øc (75 MHz; $\left.\mathrm{CDCl}_{3}\right) 205.0$ (C), 135.5 (C), 131.6 (C), 130.3 (C), 128.0 (CH), $120.4(\mathrm{CH}), 120.1(\mathrm{CH}), 112.3(\mathrm{CH}), 102.9(\mathrm{CH}), 35.3(\mathrm{CH}), 19.5(\mathrm{Me}) ; \mathrm{m} / z(\mathrm{CI}) 188$ $\left(\mathrm{MH}^{+}, 100 \%\right), 187\left(\mathrm{M}^{+}, 12\right), 144(17)$.

(b) 6-Isobutyrylindole (12.2 g, $65.2 \mathrm{mmol})$, 4-dimethylaminopyridine (238 mg, $1.95 \mathrm{mmol})$ and di-tert-butyl dicarbonate $(18.5 \mathrm{~g}, 85.0 \mathrm{mmol})$ were stirred for $15 \mathrm{~h}$ in anhydrous acetonitrile (250 $\mathrm{ml})$. The acetonitrile was removed under vacuum and the residue was dissolved in ethyl acetate $(500 \mathrm{ml})$, and was washed with saturated aqueous sodium hydrogen carbonate $(250 \mathrm{ml})$ and brine $(250 \mathrm{ml})$. The organics were dried $\left(\mathrm{MgSO}_{4}\right)$ and concentrated. The residue was purified by column chromatography ( $5 \%$ up to $15 \%$ ethyl acetate / light petroleum) to give the title compound (18.1 g, 97\%) as a colorless oil; (Found: $\mathrm{MH}^{+}, 288.1598 . \mathrm{C}_{17} \mathrm{H}_{21} \mathrm{NO}_{3}+\mathrm{H}$ requires 288.1599); $\square_{\max }\left(\right.$ film)/cm ${ }^{-1} 2975$ (ArH), 2934, 2853 (CH), 1739, 1678 (CO), 1609, 1522 (C=C); 
$\square_{\mathrm{H}}\left(300 \mathrm{MHz} ; \mathrm{CDCl}_{3}\right) 8.82(1 \mathrm{H}, \mathrm{s}, 7-\mathrm{H}), 7.89(1 \mathrm{H}, \mathrm{dd}, J 8.3,1.5,4-\mathrm{H}), 7.76(1 \mathrm{H}, \mathrm{d}, J 3.7,2-\mathrm{H})$, $7.61(1 \mathrm{H}, \mathrm{d}, J 8.3,5-\mathrm{H}), 6.62(1 \mathrm{H}, \mathrm{dd}, J 3.7,0.6,3-\mathrm{H}), 3.69\left(1 \mathrm{H}, \mathrm{m}, J 6.8, \mathrm{CHMe}_{2}\right), 1.71(9 \mathrm{H}, \mathrm{s}$, $\left.\mathrm{CMe}_{3}\right), 1.26$ (6H, d, J 6.8, $\left.\mathrm{CHMe}_{2}\right)$; $\mathrm{Zc}\left(75 \mathrm{MHz} ; \mathrm{CDCl}_{3}\right) 204.5$ (C), 149.4 (C), 134.8 (C), 134.2 (C), $132.3(\mathrm{C}), 129.1(\mathrm{CH}), 122.8(\mathrm{CH}), 120.9(\mathrm{CH}), 116.0(\mathrm{CH}), 107.2(\mathrm{CH}), 84.4(\mathrm{C}), 35.3(\mathrm{C})$, $28.1(\mathrm{Me}), 19.5(\mathrm{Me}) ; \mathrm{m} / z(\mathrm{CI}) 288\left(\mathrm{MH}^{+}, 56 \%\right), 260$ (15), 232 (100), 188 (13).

\section{Ethyl 5-(1-tert-butoxycarbonylindol-6-yl)-5-isopropyl-2-thioxo-oxazolidine-4-carboxylate 8}

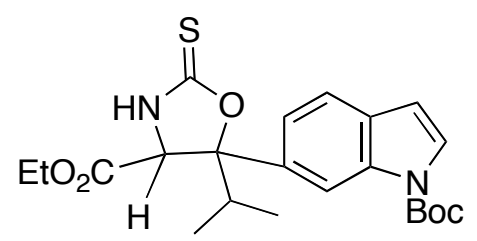

A premixed solution of $N$-tert-butoxycarbonyl-6-isobutyrylindole $7(200 \mathrm{mg}, 0.700 \mathrm{mmol})$ and ethyl isothiocyanatoacetate $(35 \square \mathrm{l}, 2.8 \mathrm{mmol})$ in anhydrous THF $(2 \mathrm{ml})$ was added dropwise to a suspension of a sodium hydride dispersion in mineral oil $(60 \% ; 120 \mathrm{mg}, 2.9 \mathrm{mmol})$ in anhydrous THF $(3 \mathrm{ml})$ at $0{ }^{\circ} \mathrm{C}$. Water $(10 \mathrm{ml})$ was added after 30 min at $0{ }^{\circ} \mathrm{C}$. The THF was removed under vacuum and the residue was dissolved in ethyl acetate $(20 \mathrm{ml})$ and washed with brine $(2 \square 10 \mathrm{ml})$. The ethyl acetate layer was dried $\left(\mathrm{MgSO}_{4}\right)$ and concentrated. The residue, containing a mixture syn- and anti-isomers, was purified and the isomers separated by column chromatography ( $30 \%$ ethyl acetate / light petroleum) to give the title compounds, anti-8 (62 mg, 21\%) as a colorless powder; mp 130-132 ${ }^{\circ} \mathrm{C}$ (from ethyl acetate / light petroleum); (Found: $\mathrm{C}, 60.7 ; \mathrm{H}, 6.5 ; \mathrm{N}, 6.5$. $\mathrm{C}_{22} \mathrm{H}_{28} \mathrm{~N}_{2} \mathrm{O}_{5} \mathrm{~S}$ requires $\mathrm{C}, 61.1 ; \mathrm{H}, 6.5 ; \mathrm{N}, 6.5 \%$ ); (Found: $\mathrm{MH}^{+}$, 433.1805. $\mathrm{C}_{22} \mathrm{H}_{28} \mathrm{~N}_{2} \mathrm{O}_{5} \mathrm{~S}+\mathrm{H}$ requires 433.1797); $\square_{\max }(\mathrm{KBr}) / \mathrm{cm}^{-1} 2978(\mathrm{CH}), 1734(\mathrm{CO}), 1207(\mathrm{CS})$; $\square_{\mathrm{H}}\left(300 \mathrm{MHz} ; \mathrm{CDCl}_{3}\right)$ $8.36(1 \mathrm{H}, \mathrm{s}, 7-\mathrm{H}), 7.80-7.65(1 \mathrm{H}, \mathrm{br} \mathrm{m}, \mathrm{NH}), 7.64(1 \mathrm{H}, \mathrm{d}, J 2.6,2-\mathrm{H}), 7.56(1 \mathrm{H}, \mathrm{d}, J 6.2,4-\mathrm{H})$, $7.41(1 \mathrm{H}, \mathrm{dd}, J 6.2,1.2,5-\mathrm{H}), 6.55(1 \mathrm{H}, \mathrm{d}, J 2.6,3-\mathrm{H}), 4.79(1 \mathrm{H}, \mathrm{s}, \mathrm{CH}), 4.39\left(2 \mathrm{H}, \mathrm{m}, \mathrm{CH}_{2} \mathrm{Me}\right)$, 
$2.66\left(1 \mathrm{H}, \mathrm{m}, J\right.$ 5.0, $\left.\underline{\mathrm{HMe}}_{2}\right), 1.69\left(9 \mathrm{H}, \mathrm{s}, \mathrm{CMe}_{3}\right), 1.42\left(3 \mathrm{H}, \mathrm{t}, J\right.$ 5.3, $\left.\mathrm{CH}_{2} \underline{\mathrm{Me}}\right), 1.03(3 \mathrm{H}, \mathrm{d}, J$ 5.0, CHMeMe), 0.77 (3H, d, J 5.0, CHMeMe); $\square \mathrm{c}\left(75 \mathrm{MHz} ; \mathrm{CDCl}_{3}\right) 188.4$ (C), 167.5 (C), 149.6 (C), $137.8(\mathrm{C}), 135.0(\mathrm{C}), 130.3(\mathrm{C}), 127.0(\mathrm{CH}), 121.1(\mathrm{CH}), 119.2(\mathrm{CH}), 111.7(\mathrm{CH}), 106.8(\mathrm{CH})$, $98.0(\mathrm{C}), 84.3(\mathrm{C}), 67.8(\mathrm{CH}), 62.8\left(\mathrm{CH}_{2}\right), 35.0(\mathrm{CH}), 28.3(\mathrm{Me}), 18.0(\mathrm{Me}), 16.6(\mathrm{Me}), 14.0$ (Me); m/z (CI) $433\left(\mathrm{MH}^{+}, 0.9 \%\right), 188$ (100), 146 (33), 118 (34), 109 (28), and syn-8 (180 mg, $60 \%$ ) as a colorless powder; $\mathrm{mp} 99-100{ }^{\circ} \mathrm{C}$ (from ethyl acetate / light petroleum); (Found: $\mathrm{C}$, 61.4; $\mathrm{H}, 7.0 ; \mathrm{N}, 6.2 . \mathrm{C}_{22} \mathrm{H}_{28} \mathrm{~N}_{2} \mathrm{O}_{5} \mathrm{~S}$ requires $\mathrm{C}, 61.1 ; \mathrm{H}, 6.5 ; \mathrm{N}, 6.5 \%$ ); (Found: $\mathrm{MH}^{+}, 433.1820$. $\mathrm{C}_{22} \mathrm{H}_{28} \mathrm{~N}_{2} \mathrm{O}_{5} \mathrm{~S}+\mathrm{H}$ requires 433.1797); $\square_{\max }(\mathrm{KBr}) / \mathrm{cm}^{-1} 2978(\mathrm{CH}), 1735(\mathrm{CO}), 1188(\mathrm{CS})$; $\mathrm{D}_{\mathrm{H}}\left(300 \mathrm{MHz} ; \mathrm{CDCl}_{3}\right) 8.14(1 \mathrm{H}, \mathrm{s}, 7-\mathrm{H}), 7.62(1 \mathrm{H}, \mathrm{d}, J 2.7,2-\mathrm{H}), 7.51(1 \mathrm{H}, \mathrm{dd}, J 6.2,0.4,4-\mathrm{H})$, $7.45(1 \mathrm{H}$, br s, NH) $7.21(1 \mathrm{H}, \mathrm{dd}, J 6.2,0.9,5-\mathrm{H}), 6.53(1 \mathrm{H}, \mathrm{d}, J 2.7,3-\mathrm{H}), 4.70(1 \mathrm{H}, \mathrm{s}, \mathrm{CH}), 3.70$ (1H, m, CㅂHMe), 3.48 (1H, m, CHMe), 2.66 (1H, m, J 5.0, $\left.\mathrm{CHMe}_{2}\right), 1.69$ (9H, s, $\left.\mathrm{CMe}_{3}\right), 1.22$ (3H, d, J 5.0, CHMeMe), 0.89 (3H, d, J 5.0, CHMeMe), 0.84 (3H, t, $J$ 5.4, $\mathrm{CH}_{2} \underline{\mathrm{Me}}$ ); $\mathrm{Zc}(75 \mathrm{MHz}$; $\left.\mathrm{CDCl}_{3}\right) 189.6(\mathrm{C}), 167.8(\mathrm{C}), 149.6(\mathrm{C}), 134.7$ (C), $132.5(\mathrm{C}), 130.5(\mathrm{C}), 127.1(\mathrm{CH}), 120.6(2$ overlapping $\mathrm{CH}), 113.0(\mathrm{CH}), 106.9(\mathrm{CH}), 98.4(\mathrm{C}), 84.3(\mathrm{C}), 66.1(\mathrm{CH}), 62.0\left(\mathrm{CH}_{2}\right), 38.0(\mathrm{CH})$, $28.2(\mathrm{Me}), 17.3(\mathrm{Me}), 16.2(\mathrm{Me}), 13.3(\mathrm{Me}) ; \mathrm{m} / z(\mathrm{CI}) 433\left(\mathrm{MH}^{+}, 0.5 \%\right), 299$ (12), 256 (12), 216 (11), $212(17), 188(100), 146(20)$.

On a larger scale, the above procedure was followed using ketone $7(18.1 \mathrm{~g}, 63.1 \mathrm{mmol})$. The isomers were purified and collected together to give the title compounds anti-8 and syn-8 $(22.5 \mathrm{~g}$, $83 \%)$.

\section{Ethyl 3-Benzyloxycarbonyl-5-(1-tert-butoxycarbonylindol-6-yl)-5-isopropyl-2-thioxo-} oxazolidine-4-carboxylate 9 


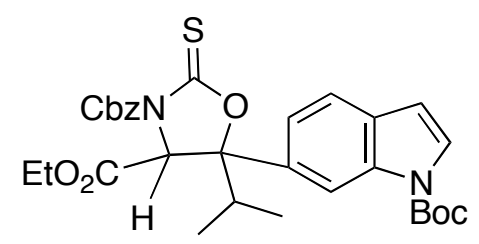

\section{(a) Anti-9}

Triethylamine (51 $\square \mathrm{l}, 0.11 \mathrm{mmol})$ and benzyl chloroformate $(51 \square 1,0.11 \mathrm{mmol})$ were respectively added dropwise to a solution of anti-8 $(47 \mathrm{mg}, 0.11 \mathrm{mmol})$ in anhydrous THF $(4 \mathrm{ml})$. The reaction mixture was stirred for $1 \mathrm{~h}$ at $0{ }^{\circ} \mathrm{C}$ and then allowed to warm to room temperature. The THF was removed under vacuum and the residue dissolved in ethyl acetate $(10 \mathrm{ml})$, and washed with water $(2 \square 10 \mathrm{ml})$. The ethyl acetate layer was dried $\left(\mathrm{MgSO}_{4}\right)$ and concentrated. The residue was purified by column chromatography ( $20 \%$ ethyl acetate / light petroleum) to give the title compound anti-9 (45 $\mathrm{mg}, 73 \%$ ) as a colorless powder; $\mathrm{mp} 156-157^{\circ} \mathrm{C}$ (from ethyl acetate / light petroleum); (Found: $\mathrm{C}, 63.5 ; \mathrm{H}, 6.1 ; \mathrm{N}, 4.9 . \mathrm{C}_{30} \mathrm{H}_{34} \mathrm{~N}_{2} \mathrm{O}_{7} \mathrm{~S}$ requires $\mathrm{C}, 63.6 ; \mathrm{H}, 6.1 ; \mathrm{N}, 5.0 \%$ ); (Found: $\mathrm{MH}^{+}$, 567.2196. $\mathrm{C}_{30} \mathrm{H}_{34} \mathrm{~N}_{2} \mathrm{O}_{7} \mathrm{~S}+\mathrm{H}$ requires 567.2165); $\square_{\max }(\mathrm{KBr}) / \mathrm{cm}^{-1} 2974(\mathrm{CH})$, 1775, $1743(\mathrm{CO})$; $\square_{\mathrm{H}}\left(300 \mathrm{MHz} ; \mathrm{CDCl}_{3}\right) 8.23(1 \mathrm{H}, \mathrm{s}, 7-\mathrm{H}), 7.69(1 \mathrm{H}, \mathrm{d}, J 3.7,2-\mathrm{H}), 7.57(1 \mathrm{H}, \mathrm{d}$, $J$ 8.3, 4-H), 7.36-7.30 (6H, m, ArH, 5-H), $6.58(1 \mathrm{H}, \mathrm{d}, J 3.7,3-\mathrm{H}), 5.30$ (2H, m, CHHAr, CH), $5.13(1 \mathrm{H}, \mathrm{d}, J 12.2, \mathrm{CH} \underline{\mathrm{HAr}}), 4.23\left(2 \mathrm{H}, \mathrm{q}, J 5.5, \underline{\mathrm{C}}_{2} \mathrm{Me}\right), 2.30\left(1 \mathrm{H}, \mathrm{m}, J 6.7, \mathrm{CHMe}_{2}\right), 1.71(9 \mathrm{H}$, s, $\left.\mathrm{CMe}_{3}\right), 1.25$ (3H, t, J 5.5, $\left.\mathrm{CH}_{2} \underline{\mathrm{Me}}\right), 0.94\left(6 \mathrm{H}, \mathrm{d}, J\right.$ 6.7, $\left.\mathrm{CHMe}_{2}\right)$; $\mathrm{Zc}\left(75 \mathrm{MHz} ; \mathrm{CDCl}_{3}\right) 182.7$ (C), 167.6 (C), 150.8 (C), 150.0 (C), 134.9 (C), 134.7 (C), 133.1 (C), 131.3 (C), $129.1(\mathrm{CH})$, 129.0 (CH), $128.8(\mathrm{CH}), 127.7(\mathrm{CH}), 121.1(\mathrm{CH}), 120.9(\mathrm{CH}), 113.8(\mathrm{CH}), 107.2(\mathrm{CH}), 94.2(\mathrm{C})$, $84.9(\mathrm{C}), 71.0(\mathrm{CH}), 69.7\left(\mathrm{CH}_{2}\right), 62.9\left(\mathrm{CH}_{2}\right), 34.2(\mathrm{CH}), 28.7(\mathrm{Me}), 18.0(\mathrm{Me}), 17.7(\mathrm{Me}), 14.3$ (Me); m/z (CI) 567 (MH+, 3\%), 423 (15), 343 (18), 301 (29), 299 (45), 258 (24), 256 (29), 232 (26), 212 (43), $188(100)$. 


\section{(b) Syn-9}

The above procedure was followed using syn-8 $(82 \mathrm{mg}, 0.19 \mathrm{mmol})$. Purification gave the title compound syn-9 (79 mg, 74\%) as a colorless powder; mp 71-74 ${ }^{\circ} \mathrm{C}$ (from ethyl acetate / light petroleum); (Found: $\mathrm{C}, 63.4 ; \mathrm{H}, 6.0 ; \mathrm{N}, 4.8 . \mathrm{C}_{30} \mathrm{H}_{34} \mathrm{~N}_{2} \mathrm{O}_{7} \mathrm{~S}$ requires $\mathrm{C}, 63.6 ; \mathrm{H}, 6.1 ; \mathrm{N}, 5.0 \%$ ); (Found: $\mathrm{MH}^{+}$, 567.2156. $\mathrm{C}_{30} \mathrm{H}_{34} \mathrm{~N}_{2} \mathrm{O}_{7} \mathrm{~S}+\mathrm{H}$ requires 567.2165); $\square_{\max }(\mathrm{KBr}) / \mathrm{cm}^{-1} 2978(\mathrm{CH})$, 1775, 1734 (CO); Пн $\left(300 \mathrm{MHz} ; \mathrm{CDCl}_{3}\right) 8.17(1 \mathrm{H}, \mathrm{s}, 7-\mathrm{H}), 7.63(1 \mathrm{H}, \mathrm{d}, J 3.6,2-\mathrm{H}), 7.50(1 \mathrm{H}, \mathrm{d}$, $J$ 8.1, 4-H), 7.45-7.31 (5H, m, ArH), $7.20(1 \mathrm{H}, \mathrm{d}, J 8.1,5-\mathrm{H}), 6.53(1 \mathrm{H}, \mathrm{d}, J 3.6,3-\mathrm{H}), 5.36(1 \mathrm{H}$, d, $J$ 12.2, CHHAr), $5.20(1 \mathrm{H}, \mathrm{d}, J 12.2, \mathrm{CH} \underline{H A r}), 5.07(1 \mathrm{H}, \mathrm{s}, \mathrm{CH}), 3.46$ (1H, m, CHHMe), 3.27

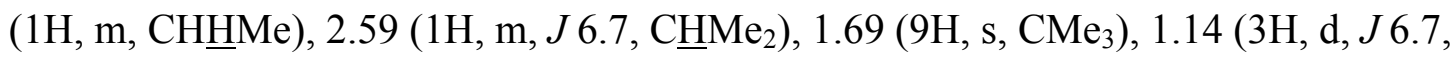
CHMeMe), 0.85 (3H, d, $J$ 6.7, CHMe $\underline{\text { Me }), ~} 0.67$ (3H, t, J 7.2, $\mathrm{CH}_{2} \underline{\mathrm{Me}}$ ); $\mathrm{Zc}\left(75 \mathrm{MHz} ; \mathrm{CDCl}_{3}\right)$ 183.2 (C), 167.1 (C), 150.5 (C), 150.0 (C), 135.0 (C), 134.7 (C), 132.2 (C), 131.0 (C), 129.1 (CH), $129.0(\mathrm{CH}), 128.8(\mathrm{CH}), 127.5(\mathrm{CH}), 120.9(\mathrm{CH}), 120.8(\mathrm{CH}), 113.5(\mathrm{CH}), 107.2(\mathrm{CH})$, $93.8(\mathrm{C}), 84.8(\mathrm{C}), 69.8\left(\mathrm{CH}_{2}\right), 69.1(\mathrm{CH}), 62.1\left(\mathrm{CH}_{2}\right), 39.5(\mathrm{CH}), 28.6(\mathrm{Me}), 17.5(\mathrm{Me}), 16.4$ (Me), $13.6(\mathrm{Me}) ; m / z(\mathrm{CI}) 567$ (MH $\left.\mathrm{MH}^{+}, 35 \%\right), 523$ (33), 511 (21), 467 (82), 423 (100), 407 (27), 300 (14), $256(24)$.

(c) On a larger scale, the above procedure was followed using a mixture of anti-8 and syn-8 (11.4 $\mathrm{g}, 26.3 \mathrm{mmol}$ ) in the same ratio in which they were formed. The isomers of the product were purified and collected together to give the title compounds anti-9 and syn-9 $(14.0 \mathrm{~g}, 94 \%)$. 
Ethyl 2-benzyloxycarbonylamino-3-(1-tert-butoxycarbonylindol-6-yl)-4-methylpent-2enoate 10

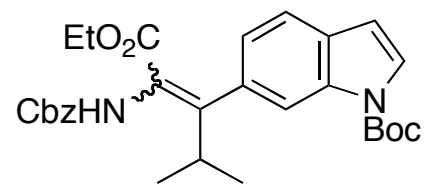

(a) From anti-N-benzyloxycarbonyl thioxo-oxazolidine 9

A solution of anti-oxazolidine 9 (43 $\mathrm{mg}, 0.08 \mathrm{mmol})$ in anhydrous THF $(0.5 \mathrm{ml})$ was added dropwise to a solution of potassium tert-butoxide (13 $\mathrm{mg}, 0.12 \mathrm{mmol})$ in anhydrous $\mathrm{THF}(1 \mathrm{ml})$ at $-78^{\circ} \mathrm{C}$. The reaction mixture was stirred for $30 \mathrm{~min}$ at this temperature and then quenched with water $(1 \mathrm{ml})$ and allowed to warm to room temperature. The THF was removed under vacuum and water $(10 \mathrm{ml})$ was added to the residue. The product was extracted with ether $(2 \square 10 \mathrm{ml})$ and the combined organics were washed with brine $(20 \mathrm{ml})$, dried $\left(\mathrm{MgSO}_{4}\right)$, and concentrated. The residue was purified and the isomers separated by column chromatography (15\% ethyl acetate / light petroleum) to give the alkenes, $(Z)-\mathbf{1 0}(8 \mathrm{mg}, 21 \%)$ as a colorless oil; (Found: $\mathrm{MH}^{+}$, 507.2491. $\mathrm{C}_{29} \mathrm{H}_{34} \mathrm{~N}_{2} \mathrm{O}_{6}+\mathrm{H}$ requires 507.2495); $\square_{\max }(\mathrm{film}) / \mathrm{cm}^{-1} 3424(\mathrm{NH}), 2976(\mathrm{CH}), 1734$ (CO); П $\square_{\mathrm{H}}\left(300 \mathrm{MHz} ; \mathrm{CDCl}_{3}\right) 7.88(1 \mathrm{H}, \mathrm{s}, 7-\mathrm{H}), 7.62(1 \mathrm{H}, \mathrm{d}, J 3.6,2-\mathrm{H}), 7.56(1 \mathrm{H}, \mathrm{d}, J$ 8.0, 4-H), 7.35-7.23 (5H, m, ArH), $6.91(1 \mathrm{H}, \mathrm{d}, J 8.0,5-\mathrm{H}), 6.57(1 \mathrm{H}, \mathrm{d}, J 3.6,3-\mathrm{H}), 5.83(1 \mathrm{H}$, br s, NH), $4.99\left(2 \mathrm{H}, \mathrm{s}, \mathrm{CH}_{2} \mathrm{Ar}\right), 4.35\left(2 \mathrm{H}, \mathrm{m}, \mathrm{CH}_{2} \mathrm{Me}\right), 3.30\left(1 \mathrm{H}, \mathrm{m}, \mathrm{C} \underline{H M e}_{2}\right), 1.66\left(9 \mathrm{H}, \mathrm{s}, \mathrm{CMe}_{3}\right), 1.35(3 \mathrm{H}$, m, $\left.\mathrm{CH}_{2} \underline{\mathrm{Me}}\right), 1.00$ (6H, d, J 6.8, $\left.\mathrm{CHMe}_{2}\right)$; $\square_{\mathrm{c}}\left(75 \mathrm{MHz} ; \mathrm{CDCl}_{3}\right) 165.5$ (C), 153.7 (C), 149.9 (C), $140.4(\mathrm{C}), 136.2(\mathrm{C}), 135.6(\mathrm{C}), 131.2(\mathrm{C}), 130.4(\mathrm{C}), 128.9(\mathrm{CH}), 128.64(\mathrm{CH}), 128.59(\mathrm{CH})$, $126.9(\mathrm{CH}), 124.7(\mathrm{C}), 123.9(\mathrm{CH}), 121.7(\mathrm{CH}), 115.7(\mathrm{CH}), 107.4(\mathrm{CH}), 84.4(\mathrm{C}), 67.6\left(\mathrm{CH}_{2}\right)$, $61.7\left(\mathrm{CH}_{2}\right), 30.7(\mathrm{CH}), 28.6(\mathrm{Me}), 21.9(\mathrm{Me}), 14.5(\mathrm{Me}) ; m / z(\mathrm{CI}) 567\left(\mathrm{MH}^{+}, 2 \%\right), 407$ (33), 361 (36), 299 (100), 298 (37), 253 (67), and (E)-10 (9 mg, 23\%), as a colorless oil; (Found: $\mathrm{M}^{+}$, 
506.2426. $\mathrm{C}_{29} \mathrm{H}_{34} \mathrm{~N}_{2} \mathrm{O}_{6}$ requires 506.2417); $\square_{\max }\left(\right.$ film) $/ \mathrm{cm}^{-1} 2975(\mathrm{CH}), 1734(\mathrm{CO})$; $\square_{\mathrm{H}}(300 \mathrm{MHz}$; $\left.\mathrm{CDCl}_{3}\right) 7.87(1 \mathrm{H}, \mathrm{s}, 7-\mathrm{H}), 7.58(1 \mathrm{H}, \mathrm{d}, J 3.6,2-\mathrm{H}), 7.47(1 \mathrm{H}, \mathrm{d}, J 8.0,4-\mathrm{H}), 7.40-7.31(5 \mathrm{H}, \mathrm{m}$, ArH), $6.96(1 \mathrm{H}, \mathrm{d}, J 8.0,5-\mathrm{H}), 6.55(1 \mathrm{H}, \mathrm{d}, J 3.6,3-\mathrm{H}), 6.25(1 \mathrm{H}, \mathrm{br} \mathrm{s}, \mathrm{NH}), 5.18\left(2 \mathrm{H}, \mathrm{s}, \mathrm{C}_{2} \mathrm{Ar}\right)$, $3.78\left(2 \mathrm{H}, \mathrm{m}, \underline{\mathrm{C}}_{2} \mathrm{Me}\right), 3.14\left(1 \mathrm{H}, \mathrm{m}, \mathrm{CHMe}_{2}\right), 1.65\left(9 \mathrm{H}, \mathrm{s}, \mathrm{CMe}_{3}\right), 1.01$ (6H, d, J 6.8, $\left.\mathrm{CHMe}_{2}\right)$, $0.66\left(3 \mathrm{H}, \mathrm{t}, J\right.$ 7.0, $\left.\mathrm{CH}_{2} \mathrm{Me}\right) ; \mathrm{Zc}_{\mathrm{c}}\left(75 \mathrm{MHz} ; \mathrm{CDCl}_{3}\right) 164.3(\mathrm{C}), 153.5(\mathrm{C}), 148.6(\mathrm{C}), 140.0(\mathrm{C})$, $135.0(\mathrm{C}), 133.5(\mathrm{C}), 131.7(\mathrm{C}), 128.5(\mathrm{C}), 127.5(\mathrm{CH}), 127.4(\mathrm{CH}), 127.2(\mathrm{CH}), 125.1(\mathrm{CH})$, $123.1(\mathrm{C}), 122.9(\mathrm{CH}), 118.6(\mathrm{CH}), 114.4(\mathrm{CH}), 106.1(\mathrm{CH}), 82.6(\mathrm{C}), 66.4\left(\mathrm{CH}_{2}\right), 59.5\left(\mathrm{CH}_{2}\right)$, $29.6(\mathrm{CH}), 27.1(\mathrm{Me}), 19.5(\mathrm{Me}), 12.3(\mathrm{Me}) ; \mathrm{m} / z$ (CI) $506\left(\mathrm{M}^{+}, 4 \%\right), 361$ (18), 343 (22), 299 (100), 298 (28), 253 (83).

(b) From syn- $N$-benzyloxycarbonyl thioxo-oxazolidine 9:

The above procedure was followed using syn-oxazolidine $9(61 \mathrm{mg}, 0.11 \mathrm{mmol})$. The isomers were purified and separated to give the alkenes $(Z)-\mathbf{1 0}(17 \mathrm{mg}, 31 \%)$, and $(E)-\mathbf{1 0}(10 \mathrm{mg}, 18 \%)$, characterized as above.

(c) On a larger scale, the above procedure was followed using a mixture of anti-10 and syn-10 $(14.0 \mathrm{~g}, 24.7 \mathrm{mmol})$ in the same ratio in which they were formed. The isomers were purified and collected together to give the alkenes $(Z)-\mathbf{1 0}$ and $(E)-\mathbf{1 0}(9.27 \mathrm{~g}, 74 \%)$.

\section{Ethyl 2-benzyloxycarbonylamino-3-(1-tert-butoxycarbonylindol-6-yl)-4-methylpentanoate}

11<smiles>CCOC(=O)NC(C(=O)OCC)C(c1ccc2ccn(C(C)(C)C)c2c1)C(C)C</smiles> 
(a) From (Z)-10

Coarse magnesium powder (47 mg, $1.98 \mathrm{mmol})$ was added to $(Z)-10(100 \mathrm{mg}, 0.198 \mathrm{mmol})$ in anhydrous methanol $(2 \mathrm{ml})$. The reaction mixture was stirred vigorously for $1 \mathrm{~h}$ ensuring the temperature did not exceed room temperature by use of an ice bath. The solvent was removed under vacuum and the residue dissolved in ethyl acetate $(20 \mathrm{ml})$ was washed with hydrochloric acid $(2 \mathrm{M} ; 15 \mathrm{ml})$ and brine $(15 \mathrm{ml})$. The organic layer was dried $\left(\mathrm{MgSO}_{4}\right)$ and concentrated. The crude product was purified and the diastereomers separated by column chromatography (5\% up to $10 \%$ ethyl acetate / light petroleum) to give diastereomer $1-( \pm)-(2 S, 3 S)-11(17 \mathrm{mg}, 17 \%)$ as a colorless oil; (Found: $\mathrm{MH}^{+}$, 509.2656. $\mathrm{C}_{29} \mathrm{H}_{36} \mathrm{~N}_{2} \mathrm{O}_{6}+\mathrm{H}$ requires 507.2651); $\square_{\max }\left(\right.$ film) $/ \mathrm{cm}^{-1} 3431$ (NH), $2978(\mathrm{CH}), 1728(\mathrm{CO})$; $\mathrm{ZH}_{\mathrm{H}}\left(400 \mathrm{MHz} ; \mathrm{CDCl}_{3}\right) 7.95(1 \mathrm{H}, \mathrm{s}, 7-\mathrm{H}), 7.55(1 \mathrm{H}, \mathrm{d}$, J 3.4, 2-H), $7.45(1 \mathrm{H}, \mathrm{d}, J 8.0,4-\mathrm{H}), 7.32(5 \mathrm{H}, \mathrm{m}, \mathrm{ArH}), 6.95(1 \mathrm{H}, \mathrm{d}, J 8.0,5-\mathrm{H}), 6.51(1 \mathrm{H}, \mathrm{d}, J 3.4,3-\mathrm{H})$, $5.13\left(3 \mathrm{H}, \mathrm{m}, \mathrm{NH}\right.$ and $\left.\underline{\mathrm{C}}_{2} \mathrm{Ar}\right), 4.97(1 \mathrm{H}, \mathrm{dd}, J 10.2,3.9, \mathrm{\square}-\mathrm{CH}), 4.12\left(2 \mathrm{H}, \mathrm{q}, J\right.$ 8.0, $\left.\underline{\mathrm{C}}_{2} \mathrm{Me}\right), 3.03$ $(1 \mathrm{H}, \mathrm{dd}, J 10.2,3.8, \square-\mathrm{CH}), 2.19\left(1 \mathrm{H}, \mathrm{m}, \mathrm{CHMe}_{2}\right), 1.63\left(9 \mathrm{H}, \mathrm{s}, \mathrm{CMe}_{3}\right), 1.25\left(6 \mathrm{H}, \mathrm{m}, \mathrm{CH}_{2} \underline{\mathrm{Me}}\right.$, CHMeMe), 0.79 (3H, d, J 6.6, CHMeMe); $\square_{c}\left(100 \mathrm{MHz} ; \mathrm{CDCl}_{3}\right) 172.0$ (C), 156.4 (C), 149.6 (C), $136.2(\mathrm{C}), 135.5(\mathrm{C}), 134.7(\mathrm{C}), 129.7(\mathrm{C}), 128.5(\mathrm{CH}), 128.14(\mathrm{CH}), 128.13(\mathrm{CH}), 126.0(\mathrm{CH})$, $123.3(\mathrm{CH}), 120.9(\mathrm{CH}), 115.3(\mathrm{CH}), 107.0(\mathrm{CH}), 83.6(\mathrm{C}), 67.1\left(\mathrm{CH}_{2}\right), 61.3\left(\mathrm{CH}_{2}\right), 55.8(\mathrm{CH})$, $55.5(\mathrm{CH}), 28.5(\mathrm{CH}), 28.1(\mathrm{Me}), 21.4(\mathrm{Me}), 21.3(\mathrm{Me}), 14.2(\mathrm{Me}) ; \mathrm{m} / z(\mathrm{CI}) 509\left(\mathrm{MH}^{+}, 27 \%\right)$, 453 (17), 409 (49), 272 (18), 244 (100), 216 (77), 158 (39), 57 (20), and diastereomer 2-(土)-(2S, 3R)-11 (52 mg, 52\%) as a colorless oil; (Found: $\mathrm{M}^{+}, 508.2567 . \mathrm{C}_{29} \mathrm{H}_{36} \mathrm{~N}_{2} \mathrm{O}_{6}$ requires 508.2573); $\square_{\max }(\mathrm{film}) / \mathrm{cm}^{-1} 3352(\mathrm{NH}), 2976(\mathrm{CH}), 1732(\mathrm{CO}) ; \square_{\mathrm{H}}\left(300 \mathrm{MHz} ; \mathrm{CDCl}_{3}\right) 7.93(1 \mathrm{H}, \mathrm{s}, 7-\mathrm{H})$, $7.54(1 \mathrm{H}, \mathrm{d}, J 3.6,2-\mathrm{H}), 7.44(1 \mathrm{H}, \mathrm{d}, J 8.1,4-\mathrm{H}), 7.33(5 \mathrm{H}, \mathrm{m}, \mathrm{ArH}), 6.91(1 \mathrm{H}, \mathrm{dd}, J$ 8.1, 1.1, 5H), $6.51(1 \mathrm{H}, \mathrm{d}, J 3.6,3-\mathrm{H}), 5.17(1 \mathrm{H}, \mathrm{m}, \mathrm{NH}), 5.12\left(2 \mathrm{H}, \mathrm{s}, \mathrm{CH}_{2} \mathrm{Ar}\right), 4.91(1 \mathrm{H}, \mathrm{m}, \mathrm{\square}-\mathrm{CH}), 4.09$ $\left(2 \mathrm{H}, \mathrm{q}, J 7.0, \mathrm{CH}_{2} \mathrm{Me}\right), 2.76(1 \mathrm{H}, \mathrm{m}, \mathrm{\square}-\mathrm{CH}), 2.38\left(1 \mathrm{H}, \mathrm{m}, \mathrm{CHMe}_{2}\right), 1.64\left(9 \mathrm{H}, \mathrm{s}, \mathrm{CMe}_{3}\right), 1.23(3 \mathrm{H}$, 
d, $J$ 6.5, CHMeMe), 1.16 (3H, t, $J$ 7.0, $\left.\mathrm{CH}_{2} \underline{\mathrm{Me}}\right), 0.77$ (3H, d, $J$ 6.5, CHMeMe); $\square \mathrm{c}(75 \mathrm{MHz}$; $\left.\mathrm{CDCl}_{3}\right)$ 171.0 (C), $155.6(\mathrm{C}), 149.6(\mathrm{C}), 136.3(\mathrm{C}), 135.3(\mathrm{C}), 134.7$ (C), 129.7 (C), $128.5(\mathrm{CH})$, $128.14(\mathrm{CH}), 128.09(\mathrm{CH}), 125.9(\mathrm{CH}), 123.8(\mathrm{CH}), 120.5(\mathrm{CH}), 115.7(\mathrm{CH}), 107.0(\mathrm{CH}), 83.5$ (C), $66.9\left(\mathrm{CH}_{2}\right), 61.2\left(\mathrm{CH}_{2}\right), 57.5(\mathrm{CH}), 55.9(\mathrm{CH}), 29.1(\mathrm{CH}), 28.2(\mathrm{Me}), 21.4(\mathrm{Me}), 21.2(\mathrm{Me})$, 13.9 (Me); m/z (FI) $509\left(\mathrm{MH}^{+}, 13 \%\right), 508\left(\mathrm{M}^{+}, 33\right) 202(21), 108$ (100).

(b) From $(E)-\mathbf{1 0}$

The above procedure was followed using $(E)-\mathbf{1 0}(60 \mathrm{mg}, 0.12 \mathrm{mmol})$. The isomers were purified and separated to give the diastereomer $1-( \pm)-(2 S, 3 S)-11(16 \mathrm{mg}, 26 \%)$ and diastereomer 2$( \pm)-(2 S, 3 R)-11(25 \mathrm{mg}, 41 \%)$, characterized as above.

(c) On a larger scale, the above procedure was followed using a mixture of $\boldsymbol{Z}-\mathbf{2 9}$ and $\boldsymbol{E}-\mathbf{2 9}$ (9.27 $\mathrm{g}, 18.3 \mathrm{mmol}$ ) in the same ratio in which they were formed. The isomers were purified and separated to give the diastereomer $1-( \pm)-(2 S, 3 S)-11(2.44 \mathrm{~g}, 26 \%)$ and diastereomer 2-( $( \pm)-(2 S$, 3R)-11 (4.01 g, 43\%), characterized as above.

\section{$( \pm)-(2 S / R, 3 R / S)$-Ethyl 2-benzyloxycarbonylamino-3-(3-formylindol-6-yl)-4-} methylpentanoate $( \pm)-12$<smiles>CCOC(=O)[C@H](c1ccc2c(C=O)c[nH]c2c1)C(C)C</smiles>

Dichloromethyl methyl ether (CAUTION) (390 $\square 1,4.27 \mathrm{mmol}$ ) was added to a solution of diastereomer $2-( \pm)-(2 S, 3 R)-11(867 \mathrm{mg}, 1.71 \mathrm{mmol})$ in dichloromethane $(10 \mathrm{ml})$ at $0{ }^{\circ} \mathrm{C}$. A 
solution of titanium tetrachloride $(670 \square \mathrm{l}, 6.15 \mathrm{mmol})$ in dichloromethane $(7 \mathrm{ml})$ was added slowly over $1 \mathrm{~h}$ to the cold mixture. The reaction mixture was kept at $0{ }^{\circ} \mathrm{C}$ for a further $5 \mathrm{~min}$ before water $(15 \mathrm{ml})$ followed by saturated aqueous sodium hydrogen carbonate $(15 \mathrm{ml})$ was added. The organic phase was separated and the aqueous was extracted with dichloromethane (3 $\square 20 \mathrm{ml})$. The combined organic layers were washed with brine $(60 \mathrm{ml})$, dried $\left(\mathrm{MgSO}_{4}\right)$ and concentrated. The residue was purified by column chromatography ( $40 \%$ up to $50 \%$ ethyl acetate / light petroleum) to give $( \pm)-\mathbf{1 2}(340 \mathrm{mg}, 46 \%)$, as a colorless powder; $\mathrm{mp} 151-153{ }^{\circ} \mathrm{C}$ (from ethyl acetate / pentane); (Found: $\mathrm{C}, 68.8 ; \mathrm{H}, 6.6 ; \mathrm{N}, 6.3 . \mathrm{C}_{25} \mathrm{H}_{28} \mathrm{~N}_{2} \mathrm{O}_{5}$ requires $\mathrm{C}, 68.8 ; \mathrm{H}, 6.5 ; \mathrm{N}$, 6.4\%); (Found: $\mathrm{M}^{+}$, 436.2007. $\mathrm{C}_{25} \mathrm{H}_{28} \mathrm{~N}_{2} \mathrm{O}_{5}$ requires 436.1998); $\square_{\max }(\mathrm{KBr}) / \mathrm{cm}^{-1} 3312(\mathrm{NH}), 2960$ (CH), 1723, 1645, $1526(\mathrm{CO})$; $\square_{\mathrm{H}}\left(300 \mathrm{MHz} ; \mathrm{CDCl}_{3}\right) 9.98(1 \mathrm{H}, \mathrm{s}, \mathrm{CHO}), 9.31(1 \mathrm{H}, \mathrm{br} \mathrm{s}, \mathrm{NH})$, $8.18(1 \mathrm{H}, \mathrm{d}, J$ 8.1, 4-H), $7.75(1 \mathrm{H}, \mathrm{d}, J 2.8,2-\mathrm{H}), 7.32(5 \mathrm{H}, \mathrm{m}, \mathrm{ArH}), 7.11(1 \mathrm{H}, \mathrm{s}, 8.2,7-\mathrm{H}), 7.00$ $(1 \mathrm{H}, \mathrm{d}, J 8.1,5-\mathrm{H}), 5.24\left(1 \mathrm{H}, \mathrm{br} \mathrm{d}, J\right.$ 8.9, NHCbz), $5.11\left(2 \mathrm{H}, \mathrm{m}, \mathrm{CH}_{2} \mathrm{Ar}\right), 4.88(1 \mathrm{H}, \mathrm{m}, \mathrm{\square}-\mathrm{CH})$, $4.07\left(2 \mathrm{H}, \mathrm{m}, \mathrm{CH}_{2} \mathrm{Me}\right), 2.78(1 \mathrm{H}, \mathrm{m}, \mathrm{\square}-\mathrm{CH}), 2.34\left(1 \mathrm{H}, \mathrm{m}, \mathrm{CHMe}_{2}\right), 1.13\left(6 \mathrm{H}, \mathrm{m}, \mathrm{CH}_{2} \mathrm{Me}\right.$,

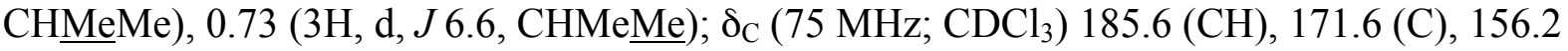
(C), $137.2(\mathrm{C}), 136.6(\mathrm{C}), 136.4(\mathrm{CH}), 135.2(\mathrm{C}), 129.0(\mathrm{CH}), 128.7(\mathrm{CH}), 128.6(\mathrm{CH}), 124.4$ $(\mathrm{CH}), 124.0(\mathrm{C}), 122.0(\mathrm{CH}), 119.7(\mathrm{C}), 112.8(\mathrm{CH}), 67.5\left(\mathrm{CH}_{2}\right), 61.8\left(\mathrm{CH}_{2}\right), 57.2(\mathrm{CH}), 56.4$ (CH), $29.4(\mathrm{CH}), 21.8(\mathrm{Me}), 21.3(\mathrm{Me}), 14.4(\mathrm{Me}) ; \mathrm{m} / z(\mathrm{EI}) 436\left(\mathrm{M}^{+}, 4 \%\right), 368$ (2), 285 (6), 212 (6), 201 (56), 200 (100), 172 (39), 158 (56), 130 (35), 98 (45), 91 (82), 80 (85), 79 (48), 55 (58). 


\section{Compound 14}
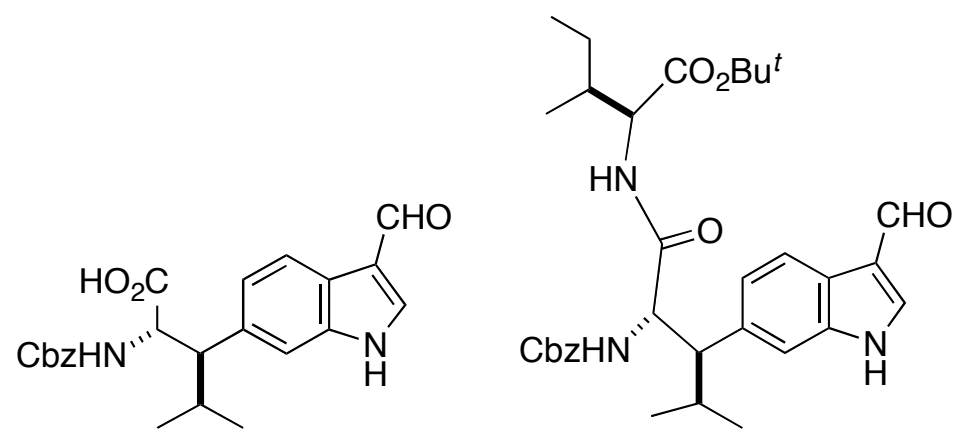

(a) Cesium carbonate $(13.0 \mathrm{~g}, 39.9 \mathrm{mmol})$ and $( \pm)-(2 S / R, 3 R / S)$-ethyl 2-benzyloxycarbonylamino3-(3-formylindol-6-yl)-4-methylpentanoate 12 (457 mg, $0.78 \mathrm{mmol})$ in water (12 ml) and methanol (12 ml) were heated under reflux for $3.5 \mathrm{~h}$. The methanol was removed under vacuum, ethyl acetate $(20 \mathrm{ml})$ and aqueous sodium hydroxide (1 M; $20 \mathrm{ml})$ were added and the layers separated. The organic layer was extracted with aqueous sodium hydroxide (1M; $20 \mathrm{ml})$ and the combined aqueous layers were acidified to $\mathrm{pH} 5$ using hydrochloric acid. The aqueous was extracted with ethyl acetate $(3 \square 30 \mathrm{ml})$ and the combined organic layers were dried $\left(\mathrm{MgSO}_{4}\right)$ and concentrated to give 2-benzyloxycarbonylamino-3-(3-formylindol-6-yl)-4-methylpentanoic acid (390 mg, 91\%) as a colorless crystalline powder; $\mathrm{mp} 150-152^{\circ} \mathrm{C}$ (from ethyl acetate / pentane); (Found: $\mathrm{C}, 67.1 ; \mathrm{H}, 6.1 ; \mathrm{N}, 6.8 . \mathrm{C}_{23} \mathrm{H}_{24} \mathrm{~N}_{2} \mathrm{O}_{5} .0 .25 \mathrm{H}_{2} \mathrm{O}$ requires $\mathrm{C}, 66.9 ; \mathrm{H}, 6.0 ; \mathrm{N}, 6.8 \%$ ); (Found: $\mathrm{MH}^{+}$, 409.1757. $\mathrm{C}_{23} \mathrm{H}_{24} \mathrm{~N}_{2} \mathrm{O}_{5}+\mathrm{H}$ requires 409.1758); $\square_{\max }(\mathrm{KBr}) / \mathrm{cm}^{-1} 3295,1573(\mathrm{NH}), 2962$ (CH), 1718, 1646, $1526(\mathrm{CO})$; $\square_{\mathrm{H}}(300 \mathrm{MHz} ; \mathrm{MeOD}) 9.74(1 \mathrm{H}, \mathrm{s}, \mathrm{CHO}), 7.94$ (2H, m, 2-H, 4-H), 7.24 (6H, m, ArH, 7-H), 6.97 (1H, d, $J 7.7,5-\mathrm{H}), 5.02(1 \mathrm{H}, \mathrm{d}, J$ 18.2, CㅂHAr), 4.98 (1H, d, $J$ 18.2, CHHAr), $4.63(1 \mathrm{H}, \mathrm{m}, \mathrm{\square}-\mathrm{CH}), 2.87(1 \mathrm{H}, \mathrm{m}, \mathrm{\square}-\mathrm{CH}), 2.26(1 \mathrm{H}, \mathrm{m}, \mathrm{CHMe}), 0.91(3 \mathrm{H}, \mathrm{d}, J$ 5.9, CHMeMe), 0.66 (3H, d, J 5.9, CHMeMe); $\square \mathrm{c}(75 \mathrm{MHz}$; MeOD) 187.8 (CH), 158.7 (C), $140.3(\mathrm{CH}), 139.3(\mathrm{C}), 138.7(\mathrm{C}), 136.9(\mathrm{C}), 129.9(\mathrm{CH}), 129.4(\mathrm{CH}), 129.2(\mathrm{CH}), 126.1(\mathrm{CH})$, $125.1(\mathrm{C}), 122.2(\mathrm{CH}), 120.4(\mathrm{C}), 114.4(\mathrm{CH}), 68.1\left(\mathrm{CH}_{2}\right), 58.3(\mathrm{CH}), 56.7(\mathrm{CH}), 30.2(\mathrm{CH}), 22.4$ 
(Me), $20.6(\mathrm{Me})$, (one quaternary carbon is not visible); $m / z(\mathrm{CI}) 409\left(\mathrm{MH}^{+}, 2 \%\right), 318(15), 132$ (25), $126(28), 108(100), 106(53), 98(45)$.

(b) Benzotriazole-1-yloxytrispyrrolidinophosphonium hexafluorophosphate (PyBOP) (642 mg, $1.28 \mathrm{mmol}$ ) was added to a solution of the above carboxylic acid (375 $\mathrm{mg}, 0.920 \mathrm{mmol})$ in dichloromethane $(9 \mathrm{ml})$ at $0{ }^{\circ} \mathrm{C}$. A premixed solution of $(S)$-isoleucine tert-butyl ester hydrochloride salt (288 mg, $1.29 \mathrm{mmol})$ and diisopropylethylamine (450 $\square 1,2.57 \mathrm{mmol})$ in dichloromethane $(9 \mathrm{ml})$ was added dropwise over $1 \mathrm{~h}$. The reaction mixture was left to warm to room temperature overnight. The solvent was removed under vacuum and the residue dissolved in ethyl acetate $(20 \mathrm{ml})$, washed with saturated aqueous sodium hydrogen carbonate $(20 \mathrm{ml})$ and brine $(20 \mathrm{ml})$, dried $\left(\mathrm{MgSO}_{4}\right)$ and concentrated. The residue was purified by column chromatography (50\% ethyl acetate / light petroleum) to give the title compound $\mathbf{1 4}$ (502 $\mathrm{mg}$, 94\%) as an inseparable 1:1 mixture of diastereomers, as a colorless sticky foam; (Found: $\mathrm{MH}^{+}$, 578.3229. $\mathrm{C}_{33} \mathrm{H}_{43} \mathrm{~N}_{3} \mathrm{O}_{6}+\mathrm{H}$ requires 578.3225); $\square \max (\mathrm{KBr}) / \mathrm{cm}^{-1} 3417(\mathrm{NH}), 2966(\mathrm{CH}), 1725$, 1652, 1529 (CO); Пн $\left(300 \mathrm{MHz} ; \mathrm{CDCl}_{3}\right)$ 10.00, 9.96 (1H, s, CHO), 9.22, 9.05 (1H, br s, NH), 8.19 (1H, m, IndH), 7.78 (1H, m, IndH), 7.35 (6H, m, IndH, 5ArH), 7.09 (1H, m, IndH), 6.41,

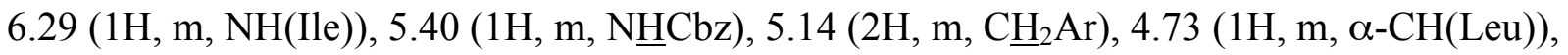
$4.17(1 \mathrm{H}, \mathrm{m}, \square-\mathrm{CH}(\mathrm{Ile})), 2.93(1 \mathrm{H}, \mathrm{m}, \square-\mathrm{CH}(\mathrm{Leu})), 2.35$ (1H, m, $\left.\underline{H M e}_{2}\right), 1.70(1 \mathrm{H}, \mathrm{m}, \mathrm{\square}-$ $\mathrm{CH}(\mathrm{Ile})), 1.39,1.25$ (9H, s, $\left.\mathrm{CMe}_{3}\right), 0.97-0.75,0.68,0.50,0.18\left(14 \mathrm{H}, \mathrm{m}, 4 \square \mathrm{Me}, \mathrm{CH}_{2} \mathrm{Me}\right)$; $m / z(\mathrm{CI}) 578\left(\mathrm{MH}^{+},<1 \%\right), 188$ (11), 140 (20), 128 (37), 114 (56), 112 (71), 100 (92), 86 (100), $72(68)$.

\section{Dehydrotryptophans 15}



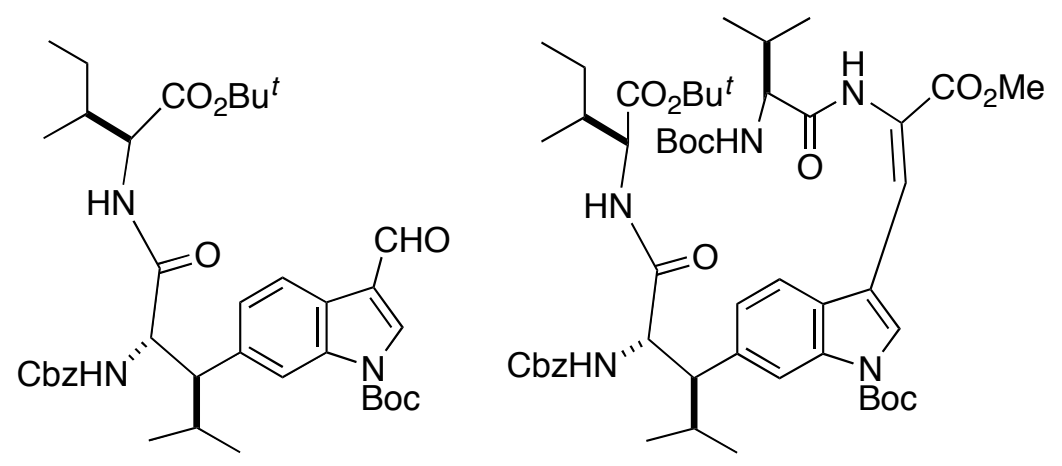

(a) Di-tert-butyl dicarbonate $(208 \mathrm{mg}, 0.960 \mathrm{mmol})$ in acetonitrile $(10 \mathrm{ml})$ was added slowly over $1 \mathrm{~h}$ to a solution $14(502 \mathrm{mg}, 0.870 \mathrm{mmol})$ and 4-dimethylaminopyridine (11 $\mathrm{mg}, 0.090 \mathrm{mmol})$ in acetonitrile $(10 \mathrm{ml})$. The reaction mixture was stirred for $3 \mathrm{~h}$ and the solvent removed under vacuum. The residue was dissolved in ethyl acetate $(30 \mathrm{ml})$, washed with saturated aqueous sodium hydrogen carbonate $(30 \mathrm{ml})$ and brine $(30 \mathrm{ml})$, dried $\left(\mathrm{MgSO}_{4}\right)$ and concentrated. The residue was purified by column chromatography (50\% ethyl acetate / light petroleum) to give $\mathrm{N}$ Boc-14 (445 mg, 76\%) as an inseparable 1:1 mixture of diastereomers, as a colorless sticky foam; (Found: $\mathrm{M}^{+}$, 677.3665. $\mathrm{C}_{38} \mathrm{H}_{51} \mathrm{~N}_{3} \mathrm{O}_{8}$ requires 677.3676); $\square_{\max }(\mathrm{KBr}) / \mathrm{cm}^{-1} 3350(\mathrm{NH}), 2967(\mathrm{CH})$, 1735, 1678, 1548, 1520 (CO); Пн (300 MHz; $\left.\mathrm{CDCl}_{3}\right) 10.06$ (1H, s, CHO), 8.20, (2H, m, IndH), $8.00(1 \mathrm{H}, \mathrm{m}, \mathrm{IndH}), 7.34$ (5H, m, ArH), 7.16 (1H, m, IndH), 6.30, 6.17 (1H, m, NH(Ile)), 5.28 (1H, m, N $\underline{H C b z}), 5.15\left(2 \mathrm{H}, \mathrm{m}, \mathrm{CH}_{2} \mathrm{Ar}\right), 4.74(1 \mathrm{H}, \mathrm{m}, \square-\mathrm{CH}(\mathrm{Leu})), 4.20$ (1H, m, $\left.\square-\mathrm{CH}(\mathrm{Ile})\right), 2.95$

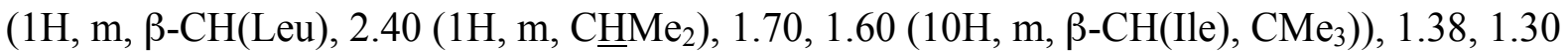
(9H, s, $\left.\mathrm{CMe}_{3}\right), 1.10\left(2 \mathrm{H}, \mathrm{m}, \mathrm{CH}_{2} \mathrm{Me}\right), 1.03,0.92(3 \mathrm{H}, \mathrm{m}, \mathrm{Me}), 0.84,0.70(6 \mathrm{H}, \mathrm{m}, \mathrm{Me}), 0.61,0.29$ (3H, m, Me); m/z (EI) 677 (M+ , <1\%), 647 (8), 526 (3), 466 (12), 244 (43), 200 (100), 158 (923), $111(29), 97(50)$.

(a) The above indole-3-carboxaldehyde $14(437 \mathrm{mg}, 0.645 \mathrm{mmol})$ in dichloromethane $(8 \mathrm{ml})$ was added dropwise to a solution of phosphonoglycine $\mathbf{1 3}^{2}(460 \mathrm{mg}, 1.16 \mathrm{mmol})$ and distilled 
1,8-diazabicyclo[5.4.0]undec-7-ene $(190 \square \mathrm{l}, 1.26 \mathrm{mmol})$ in dichloromethane $(8 \mathrm{ml})$ at $0{ }^{\circ} \mathrm{C}$. The reaction mixture was allowed to warm to room temperature overnight, and the solvent removed under vacuum. The residue was dissolved in ethyl acetate $(20 \mathrm{ml})$, washed with saturated aqueous sodium hydrogen carbonate $(10 \mathrm{ml})$ and brine $(10 \mathrm{ml})$, and the organic layer dried $\left(\mathrm{MgSO}_{4}\right)$ and concentrated. The residue was purified and the diastereomers separated by column chromatography (20\% up to $30 \%$ ethyl acetate / light petroleum) to give the dehydrotryptophans D1-15 (207 mg, 34\%) as a colorless powder; $\mathrm{mp} 111-112{ }^{\circ} \mathrm{C}$ (from ethyl acetate / pentane); (Found: $\mathrm{C}, 64.2 ; \mathrm{H}, 7.9 ; \mathrm{N}, 7.1 . \mathrm{C}_{51} \mathrm{H}_{73} \mathrm{~N}_{5} \mathrm{O}_{12} .0 .33 \mathrm{H}_{2} \mathrm{O}$ requires $\mathrm{C}, 64.2 ; \mathrm{H}, 7.8 ; \mathrm{N}, 7.3 \%$ ); (Found: $\mathrm{MH}^{+}$, 948.5322. $\mathrm{C}_{51} \mathrm{H}_{73} \mathrm{~N}_{5} \mathrm{O}_{12}+\mathrm{H}$ requires 948.5328); $\square_{\max }(\mathrm{KBr}) / \mathrm{cm}^{-1} 3348(\mathrm{NH}), 2969$ (CH), 1728, 1693 (CO), 1499 (Aryl CC); $\square_{\mathrm{H}}\left(400 \mathrm{MHz} ; \mathrm{CDCl}_{3}\right) 7.91(1 \mathrm{H}, \mathrm{s}, \mathrm{NH}), 7.86(1 \mathrm{H}, \mathrm{s}, 7-$ H), $7.70(1 \mathrm{H}, \mathrm{s}, 2-\mathrm{H}), 7.42(2 \mathrm{H}, \mathrm{m},=\mathrm{CH}, 4-\mathrm{H}$ or $5-\mathrm{H}), 7.33(5 \mathrm{H}, \mathrm{m}, \mathrm{ArH}), 7.16(1 \mathrm{H}, \mathrm{d}, J 7.9,4-\mathrm{H}$ or 5-H), $6.90(1 \mathrm{H}$, br d, $J 7.7, \mathrm{NH}(\mathrm{Ile})), 5.38(1 \mathrm{H}$, br d, $J 9.1, \mathrm{~N} \underline{\mathrm{HCbz}}), 5.23$ (1H, br d, $J 9.6$, N $\underline{H B o c}), 5.13\left(2 \mathrm{H}, \mathrm{m}, \mathrm{CH}_{2} \mathrm{Ar}\right), 4.70(1 \mathrm{H}, \mathrm{m}, \mathrm{\square}-\mathrm{CH}(\mathrm{Leu})), 4.40(1 \mathrm{H}, \mathrm{m}, \mathrm{\square}-\mathrm{CH}(\mathrm{Val})), 4.06(1 \mathrm{H}$, m, $\square-\mathrm{CH}(\mathrm{Ile})), 3.84(3 \mathrm{H}, \mathrm{s}, \mathrm{OMe}), 2.87(1 \mathrm{H}, \mathrm{m}, \mathrm{\square}-\mathrm{CH}(\mathrm{Leu})), 2.32(1 \mathrm{H}, \mathrm{m}, \mathrm{\square}-\mathrm{CH}(\mathrm{Val})), 2.07(1 \mathrm{H}$, m, $\left.\underline{\mathrm{HMe}}_{2}\right), 1.66\left(10 \mathrm{H}, \mathrm{m}, \mathrm{CMe}_{3}, \mathrm{\square}-\mathrm{CH}(\mathrm{Ile})\right), 1.54\left(9 \mathrm{H}, \mathrm{s}, \mathrm{CMe}_{3}\right), 1.40\left(2 \mathrm{H}, \mathrm{m}, \mathrm{C}_{2} \mathrm{Me}\right), 1.11$ (9H, s, $\left.\mathrm{CMe}_{3}\right), 0.99(3 \mathrm{H}, \mathrm{d}, J 6.8, \mathrm{Me}(\mathrm{Leu})), 0.95(3 \mathrm{H}, \mathrm{d}, J 6.8, \mathrm{Me}(\mathrm{Leu})), 0.88$ (6H, m, $\left.(\mathrm{Me})_{2}(\mathrm{Val})\right), 0.78\left(3 \mathrm{H}, \mathrm{t}, J\right.$ 7.3, $\left.\mathrm{CH}_{2} \underline{\mathrm{Me}}\right), 0.72(3 \mathrm{H}, \mathrm{d}, J 6.8, \mathrm{CHMe}(\mathrm{Ile})) ; \mathrm{Zc}\left(100 \mathrm{MHz} ; \mathrm{CDCl}_{3}\right)$ 171.6 (C), 171.3 (C), 170.8 (C), 169.9 (C), 165.1 (C), 155.7 (C), 148.9 (C), 136.5 (C), 135.3 (C), $134.7(\mathrm{C}), 128.5(\mathrm{CH}), 128.03(\mathrm{CH}), 127.96(\mathrm{CH}), 127.5(\mathrm{CH}), 126.7(\mathrm{C}), 125.1(\mathrm{C}), 124.1(\mathrm{CH})$, $122.7(\mathrm{CH}), 120.6(\mathrm{CH}), 117.3(\mathrm{CH}), 114.1(\mathrm{CH}), 84.4(\mathrm{C}), 81.5(\mathrm{C}), 80.2(\mathrm{C}), 66.8\left(\mathrm{CH}_{2}\right), 58.9$ $(\mathrm{CH}), 57.1(\mathrm{CH}), 56.9(\mathrm{CH}), 55.5(\mathrm{CH}), 52.4(\mathrm{Me}), 37.2(\mathrm{CH}), 31.7(\mathrm{CH}), 28.5(\mathrm{Me}), 28.3(\mathrm{CH})$, $28.1(\mathrm{Me}), 27.6(\mathrm{Me}), 25.3\left(\mathrm{CH}_{2}\right), 21.8(\mathrm{Me}), 18.8(\mathrm{Me}), 18.0(2$ overlapping $\mathrm{Me}), 15.1(\mathrm{Me})$, $11.0(\mathrm{Me}) ; \mathrm{m} / z(\mathrm{ES}) 971\left(\mathrm{M}+\mathrm{Na}^{+}, 100 \%\right), 949\left(\mathrm{M}+\mathrm{H}^{+}, 91\right), 849$ (99), 792 (52), 91 (23), and D2- 
15 (237 mg, 39\%) as a colorless powder; mp $124-126^{\circ} \mathrm{C}$ (from ethyl acetate / pentane); (Found: C, 63.9; $\mathrm{H}, 8.0 ; \mathrm{N}$, 7.2. $\mathrm{C}_{51} \mathrm{H}_{73} \mathrm{~N}_{5} \mathrm{O}_{12} .0 .5 \mathrm{H}_{2} \mathrm{O}$ requires $\mathrm{C}, 64.0 ; \mathrm{H}, 7.8 ; \mathrm{N}, 7.3 \%$ ); (Found: $\mathrm{MH}^{+}$, 948.5327. $\mathrm{C}_{51} \mathrm{H}_{73} \mathrm{~N}_{5} \mathrm{O}_{12}+\mathrm{H}$ requires 948.5328); $\square_{\max }(\mathrm{KBr}) / \mathrm{cm}^{-1} 3337(\mathrm{NH}), 2968(\mathrm{CH}), 1728$ (CO), 1506 (Aryl CC); $\square_{\mathrm{H}}\left(400 \mathrm{MHz} ; \mathrm{CDCl}_{3}\right) 7.99$ (1H, s, 7-H), $7.92(1 \mathrm{H}, \mathrm{s}, 2-\mathrm{H}), 7.63(1 \mathrm{H}, \mathrm{s}$, $=\mathrm{CH}), 7.60(1 \mathrm{H}, \mathrm{s}, \mathrm{NH}), 7.56(1 \mathrm{H}, \mathrm{d}, J 8.0,4-\mathrm{H}$, or 5-H), $7.33(5 \mathrm{H}, \mathrm{m}, \mathrm{ArH}), 7.12(1 \mathrm{H}, \mathrm{d}, J 8.0$, 4-H or 5-H), $6.15(1 \mathrm{H}$, br d, $J 6.5, \mathrm{NH}(\mathrm{Ile}$ or $\mathrm{Val})), 5.37(1 \mathrm{H}, \mathrm{d}, J 9.0, \mathrm{~N} \underline{\mathrm{HCbz}}), 5.13(2 \mathrm{H}, \mathrm{m}$, $\left.\mathrm{CH}_{2} \mathrm{Ar}\right), 5.07(1 \mathrm{H}, \mathrm{d}, J$ 9.2, NH(Ile or Val)), $4.72(1 \mathrm{H}, \mathrm{m}, \mathrm{\square}-\mathrm{CH}(\mathrm{Leu})), 4.19(1 \mathrm{H}, \mathrm{m}, \mathrm{\square}-\mathrm{CH}(\mathrm{Ile}$ or Val)), $4.12(1 \mathrm{H}, \mathrm{m}, \mathrm{C}-\mathrm{CH}(\mathrm{Ile}$ or Val)), 3.85 (3H, s, OMe), 2.97 (1H, m, $\mathrm{\square}-\mathrm{CH}(\mathrm{Leu})), 2.35(2 \mathrm{H}$,

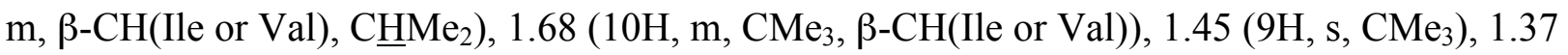
(9H, s, $\left.\mathrm{CMe}_{3}\right), 1.31\left(2 \mathrm{H}, \mathrm{m}, \mathrm{CH}_{2} \mathrm{Me}\right), 1.03(3 \mathrm{H}, \mathrm{d}, J 6.8, \mathrm{Me}), 0.99$ (3H, d, J 6.8, Me), 0.90 (3H, d, $J 6.8, \mathrm{Me}), 0.86(3 \mathrm{H}, \mathrm{d}, J 6.8, \mathrm{Me}), 0.58(3 \mathrm{H}, \mathrm{d}, J 6.8, \mathrm{Me}), 0.26(3 \mathrm{H}, \mathrm{d}, J 6.8, \mathrm{Me}(\mathrm{Ile}))$; $\mathrm{Zc}$ (100 MHz; $\left.\mathrm{CDCl}_{3}\right)$ 170.38 (C), 170.37 (C), 170.3 (C), 170.1 (C), 165.3 (C), 156.0 (C), 149.0 (C), $136.2(\mathrm{C}), 135.3(\mathrm{C}), 134.9(\mathrm{C}), 128.7(\mathrm{C}), 128.5(\mathrm{CH}), 128.13(\mathrm{CH}), 128.07(\mathrm{CH}), 127.6(\mathrm{CH})$, $125.2(\mathrm{CH}), 123.8(\mathrm{CH}), 123.1(\mathrm{C}), 118.5(\mathrm{CH}), 116.2(\mathrm{CH}), 113.7(\mathrm{C}), 84.7(\mathrm{C}), 81.9(\mathrm{C}), 80.2$ (C), $67.1\left(\mathrm{CH}_{2}\right), 61.8(\mathrm{CH}), 57.1(\mathrm{CH}), 56.6(\mathrm{CH}), 54.9(\mathrm{CH}), 52.6(\mathrm{Me}), 38.0(\mathrm{CH}), 30.2(\mathrm{CH})$, $28.3(\mathrm{Me}), 28.2(\mathrm{CH}), 28.1(\mathrm{Me}), 28.0(\mathrm{Me}), 24.7\left(\mathrm{CH}_{2}\right), 21.7(\mathrm{Me}), 19.5(\mathrm{Me}), 18.4(\mathrm{Me}), 17.5$ (Me), $14.5(\mathrm{Me}), 11.4(\mathrm{Me}) ; \mathrm{m} / z$ (ES) $971\left(\mathrm{M}+\mathrm{Na}^{+}, 100 \%\right), 949\left(\mathrm{M}+\mathrm{H}^{+}, 20\right), 849$ (31), 793 (14), $91(12), 46(20)$.

\section{Compounds 16}




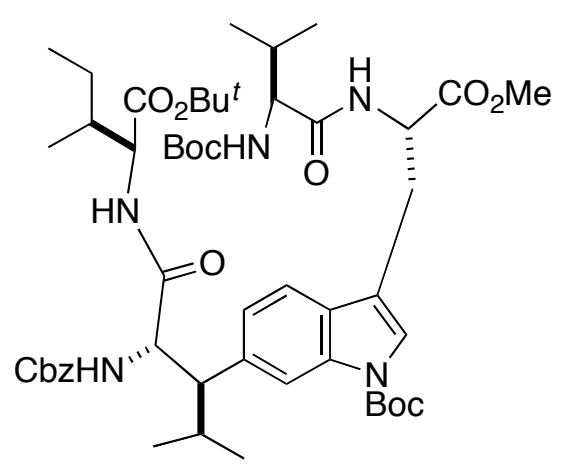

(a) D1-16

Anhydrous methanol (12 ml) was added to dehydrotryptophan D1-15 (164 mg, $0.173 \mathrm{mmol})$ and (+)-1,2-bis((2S,5S)-2,5-diethylphospholano)benzene (cyclooctadiene) rhodium(I) trifluoromethanesulfonate $(6.5 \mathrm{mg}, 5 \mathrm{~mol} \%)$ contained in a dry Parr tube. The system was evacuated and flushed with nitrogen 5 times, and evacuated and flushed with hydrogen 5 times. The hydrogen pressure was increased to $90 \mathrm{psi}$. The reaction mixture was left to stir for $42 \mathrm{~h}$ and the solvent was removed under vacuum. The residue was purified by column chromatography (30\% ethyl acetate / light petroleum) to give D1-16 (161 mg, 98\%, 99\% de) as colorless crystals; mp 107-109 ${ }^{\circ} \mathrm{C}$ (from ethyl acetate / pentane); $[\square]_{D}^{30} 28.6$ (c 0.42, $\mathrm{CHCl}_{3}$ ) (Found: $\mathrm{MH}^{+}$, 950.5489. $\mathrm{C}_{51} \mathrm{H}_{75} \mathrm{~N}_{5} \mathrm{O}_{12}+\mathrm{H}$ requires 950.5485); $\square_{\max }(\mathrm{KBr}) / \mathrm{cm}^{-1} 3371(\mathrm{NH}), 2968,2934,2876$ (CH), 1732, $1668(\mathrm{CO}), 1505$ (Aryl CC); П $\mathrm{H}_{\mathrm{H}}\left(400 \mathrm{MHz} ; \mathrm{CDCl}_{3}\right) 7.73(1 \mathrm{H}, \mathrm{s}, 7-\mathrm{H}), 7.32(6 \mathrm{H}, \mathrm{m}$, ArH, 4-H or 5-H), $7.23(1 \mathrm{H}, \mathrm{s}, 2-\mathrm{H}), 7.11(1 \mathrm{H}, \mathrm{d}, J 7.8,4-\mathrm{H}$ or 5-H), $6.98(1 \mathrm{H}, \mathrm{d}, J$ 9.2, NH(Ile)),

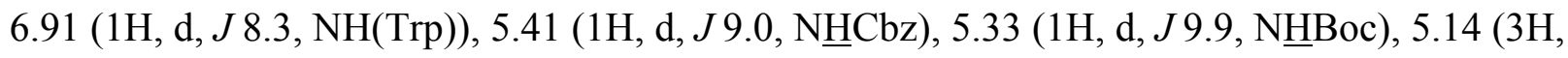
m, $\left.\underline{\mathrm{C}}_{2} \mathrm{Ar}, \square-\mathrm{CH}(\mathrm{Trp})\right), 4.69(1 \mathrm{H}, \mathrm{m}, \square-\mathrm{CH}(\mathrm{Leu})), 4.33(1 \mathrm{H}, \mathrm{m}, \mathrm{\square}-\mathrm{CH}(\mathrm{Val})), 4.02(1 \mathrm{H}, \mathrm{m}, \mathrm{\square}-$ $\mathrm{CH}(\mathrm{Ile})), 3.75$ (3H, s, OMe), 3.29 (2H, m, $\left.\square-\mathrm{CH}_{2}(\mathrm{Trp})\right), 2.86(1 \mathrm{H}, \mathrm{m}, \mathrm{\square}-\mathrm{CH}(\mathrm{Leu})), 2.28(1 \mathrm{H}, \mathrm{m}$, $\left.\mathrm{CHMe}_{2}\right), 1.86(1 \mathrm{H}, \mathrm{m}, \mathrm{\square}-\mathrm{CH}(\mathrm{Val})), 1.77\left(1 \mathrm{H}, \mathrm{m}, \mathrm{\square}-\mathrm{CH}(\mathrm{Ile}), 1.63\left(9 \mathrm{H}, \mathrm{s}, \mathrm{CMe}_{3}\right), 1.59\right.$ (9H, s, $\left.\mathrm{CMe}_{3}\right), 1.46(1 \mathrm{H}, \mathrm{m}, \mathrm{CH} \underline{\mathrm{HMe}}), 1.11\left(9 \mathrm{H}, \mathrm{s}, \mathrm{CMe}_{3}\right), 1.01(1 \mathrm{H}, \mathrm{m}, \mathrm{C} \underline{\mathrm{HHMe}}), 0.85(9 \mathrm{H}, \mathrm{m}, 3 \mathrm{\square}$ $\mathrm{Me}), 0.75$ (9H, m, $3 \square \mathrm{Me})$; $\square_{\mathrm{c}}\left(100 \mathrm{MHz} ; \mathrm{CDCl}_{3}\right) 171.8(\mathrm{C}), 171.6(\mathrm{C}), 171.2(\mathrm{C}), 171.0(\mathrm{C})$, 
155.6 (C), 155.4 (C), 149.3 (C), $136.6(\mathrm{C}), 135.0$ (C), 133.8 (C), $130.0(\mathrm{C}), 128.4(\mathrm{CH}), 128.0$ (CH), $127.9(\mathrm{CH}), 123.4(2$ overlapping $\mathrm{CH}), 118.7(\mathrm{CH}), 117.5(\mathrm{CH}), 115.2(\mathrm{C}), 83.4(\mathrm{C}), 81.3$ (C), $80.0(\mathrm{C}), 66.7\left(\mathrm{CH}_{2}\right), 58.4(\mathrm{CH}), 57.3(\mathrm{CH}), 56.9(\mathrm{CH}), 54.4(\mathrm{CH}), 53.0(\mathrm{CH}), 52.2(\mathrm{Me})$, 36.2 (CH), $32.5(\mathrm{CH}), 28.6(\mathrm{Me}), 28.2(\mathrm{Me}), 27.9(\mathrm{CH}), 27.3(\mathrm{Me}), 26.7\left(\mathrm{CH}_{2}\right), 25.1\left(\mathrm{CH}_{2}\right), 21.8$ (Me), $18.6(\mathrm{Me}), 18.3(\mathrm{Me}), 17.4(\mathrm{Me}), 15.1(\mathrm{Me}), 10.7(\mathrm{Me}) ; m / z(\mathrm{ES}) 973\left(\mathrm{M}^{+\mathrm{Na}^{+}}, 100 \%\right), 951$ $\left(\mathrm{M}+\mathrm{H}^{+}, 38\right), 851$ (72), 164 (13), 91 (15).

\section{(b) D2-16}

An identical procedure to above was followed using dehydrotryptophan D2-15 (161 mg, $0.170 \mathrm{mmol}$ ). Purification gave D2-16 (162 mg, $>99 \%,>99 \% \mathrm{de}$ ) as colorless crystals; mp 94-96 ${ }^{\circ} \mathrm{C}$ (from ethyl acetate / pentane); $[\square]_{D}^{30} 23.5$ ( $c 0.51, \mathrm{CHCl}_{3}$ ) (Found: $\mathrm{MH}^{+}, 950.5478$. $\mathrm{C}_{51} \mathrm{H}_{75} \mathrm{~N}_{5} \mathrm{O}_{12}+\mathrm{H}$ requires 950.5485); $\square_{\max }(\mathrm{KBr}) / \mathrm{cm}^{-1} 3427,3328(\mathrm{NH}), 2968,2934,2876(\mathrm{CH})$, 1733, 1678 (CO), 1511 (Aryl CC); $\square_{\mathrm{H}}\left(400 \mathrm{MHz} ; \mathrm{CDCl}_{3}\right) 7.95(1 \mathrm{H}, \mathrm{s}, 7-\mathrm{H}), 7.33$ (7H, m, 5ArH, 2-H, 4-H or 5-H), $7.05(1 \mathrm{H}, \mathrm{d}, J$ 7.7, 4-H or 5-H), $6.67(1 \mathrm{H}, \mathrm{br}$ s, $\mathrm{NH}(\mathrm{Trp})), 6.20(1 \mathrm{H}, \mathrm{d}, J$ 7.5,

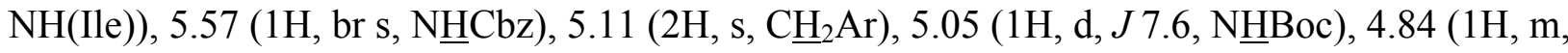

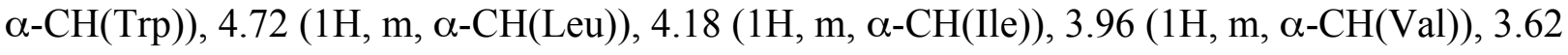
(3H, s, OMe), $3.13\left(2 \mathrm{H}, \mathrm{m}, \square-\mathrm{CH}_{2}(\mathrm{Trp})\right), 2.96(1 \mathrm{H}, \mathrm{m}, \mathrm{\square}-\mathrm{CH}(\mathrm{Leu})), 2.33\left(1 \mathrm{H}, \mathrm{m}, \mathrm{CHMe}_{2}\right), 2.09$ $(1 \mathrm{H}, \mathrm{m}, \mathrm{\square}-\mathrm{CH}(\mathrm{Val})), 1.65\left(9 \mathrm{H}, \mathrm{s}, \mathrm{CMe}_{3}\right), 1.40\left(9 \mathrm{H}, \mathrm{s}, \mathrm{CMe}_{3}\right), 1.36\left(9 \mathrm{H}, \mathrm{s}, \mathrm{CMe}_{3}\right), 1.30(1 \mathrm{H}, \mathrm{m}, \textrm{ }-$ CH(Ile)), 1.00 (1H, m, CㅂHMe), 0.87 (12H, m, $2 \square \mathrm{Me}(\mathrm{Val}), 2 \square \mathrm{Me}(\mathrm{Leu})), 0.61(1 \mathrm{H}, \mathrm{m}$, CHEMe), 0.55 (3H, m, $\left.\mathrm{CH}_{2} \mathrm{Me}\right), 0.22$ (3H, d J 6.0, $\left.\mathrm{CHMe}\right)$; $\mathrm{Zc}\left(100 \mathrm{MHz} ; \mathrm{CDCl}_{3}\right) 171.8$ (C), 171.5 (C), 170.4 (C), 170.1 (C), 156.0 (C), 155.7 (C), 149.3 (C), 136.3 (C), 134.63 (C), 134.60 (C), $129.4(\mathrm{C}), 128.5(\mathrm{CH}), 128.1(\mathrm{CH}), 128.0(\mathrm{CH}), 124.8(\mathrm{CH}), 124.0(\mathrm{CH}), 118.4(\mathrm{CH}), 116.1$ $(\mathrm{CH}), 114.7(\mathrm{C}), 83.8(\mathrm{C}), 81.8(\mathrm{C}), 79.9(\mathrm{C}), 67.0\left(\mathrm{CH}_{2}\right), 59.7(\mathrm{CH}), 57.0(\mathrm{CH}), 56.6(\mathrm{CH}), 54.6$ 
(CH), 52.4 (overlapping $\mathrm{CH}$ and $\mathrm{Me}$ ), $37.9(\mathrm{CH}), 30.9(\mathrm{CH}), 28.25(\mathrm{Me}), 28.19$ (overlapping $\mathrm{CH}$ and $\mathrm{Me}), 27.9(\mathrm{Me}), 27.6\left(\mathrm{CH}_{2}\right), 24.6\left(\mathrm{CH}_{2}\right), 21.7(\mathrm{Me}), 19.2(\mathrm{Me}), 18.3(\mathrm{Me}), 17.6(\mathrm{Me}), 14.4$ (Me), $11.4(\mathrm{Me}) ; m / z(\mathrm{ES}) 973\left(\mathrm{M}+\mathrm{Na}^{+}, 100 \%\right), 951\left(\mathrm{M}+\mathrm{H}^{+}, 15\right), 851(78), 795$ (11), 91 (8).

(c) Achiral hydrogenation using D1-15

Anhydrous methanol (1 ml) was added to dehydrotryptophan D1-15 (9 mg, $0.009 \mathrm{mmol})$ and 1,1'-bis(di-iso-propylphosphino)ferrocene (1,5-cyclooctadiene) rhodium(I) tetrafluoroborate (1.5 mg, 2 mol\%) contained in a dry Parr tube. The system was evacuated and flushed with nitrogen 5 times, and evacuated and flushed with hydrogen 5 times. The hydrogen pressure was increased to 90 psi. The reaction mixture was left to stir for $20 \mathrm{~h}$ and the solvent was removed under vacuum. The residue was purified by column chromatography (30\% ethyl acetate / light petroleum) to give a 1:1 mixture of diastereomers (9 $\mathrm{mg}, 100 \%)$ as a colorless powder.

(d) Achiral hydrogenation using using D2-15

An identical procedure to above was followed using dehydrotryptophan D2-15 (8 $\mathrm{mg}$, $0.008 \mathrm{mmol})$. Purification gave a 1:1 mixture of diastereomers ( $8 \mathrm{mg}, 100 \%)$ as a colorless powder.

\section{Compounds 17}

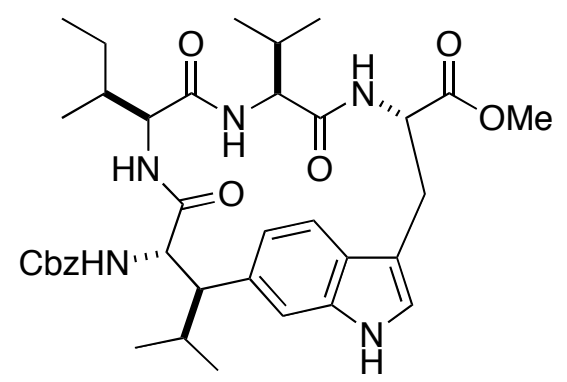




\section{(a) D1-17}

Trifluoroacetic acid $(0.7 \mathrm{ml})$ was added slowly to a solution of D1-16 (43 $\mathrm{mg}, 0.045 \mathrm{mmol})$ in dichloromethane $(0.7 \mathrm{ml})$ at room temperature. After $2.5 \mathrm{~h}$ the reaction mixture was concentrated ensuring all excess trifluoroacetic had been removed. The residue was dissolved in anhydrous DMF (45 ml), cooled to $0{ }^{\circ} \mathrm{C}$ and HOAt (47 mg, $\left.0.344 \mathrm{mmol}\right)$, diisopropylethylamine (90 $\square$, $0.52 \mathrm{mmol})$ and HATU (65 $\mathrm{mg}, 0.17 \mathrm{mmol})$ were added sequentially. The reaction mixture was allowed to warm to room temperature and, after stirring for 4 days, was concentrated. The residue was dissolved in ethyl acetate $(20 \mathrm{ml})$ and was washed with hydrochloric acid $(1 \mathrm{M} ; 10 \mathrm{ml})$, saturated aqueous sodium hydrogen carbonate $(2 \square 10 \mathrm{ml})$ and brine $(10 \mathrm{ml})$. The organic layer was dried $\left(\mathrm{MgSO}_{4}\right)$ and concentrated. The residue was purified by column chromatography $(50 \%$ up to $80 \%$ ethyl acetate) to give D1-17 (15 mg, 49\%) as a colorless solid; (Found: $\mathrm{MH}^{+}$, 676.3698. $\mathrm{C}_{37} \mathrm{H}_{49} \mathrm{~N}_{5} \mathrm{O}_{7}+\mathrm{H}$ requires 676.3705); $\square_{\mathrm{H}}(400 \mathrm{MHz}$; MeOD) $7.38(1 \mathrm{H}, \mathrm{d}, J$ 8.9, 4-H), 7.22 (5H, m, ArH), 6.85 (3H, m, 2-H, 5-H, 7-H), 5.36 (1H, m, $\square-\mathrm{CH}(\operatorname{Trp})), 5.00\left(2 \mathrm{H}, \mathrm{m}_{,} \mathrm{C}_{2} \mathrm{Ar}\right)$, $4.52(1 \mathrm{H}, \mathrm{m}, \square-\mathrm{CH}(\mathrm{Leu})), 3.86(1 \mathrm{H}, \mathrm{d}, J 8.4, \square-\mathrm{CH}(\mathrm{Ile}$ or Val)), $3.76(1 \mathrm{H}, \mathrm{d}, J 6.6, \square-\mathrm{CH}(\mathrm{Ile}$ or

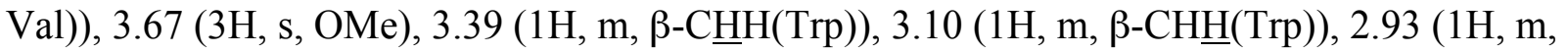

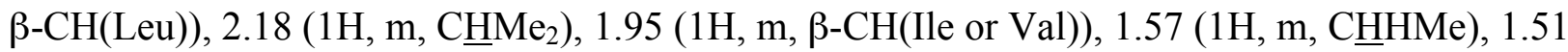

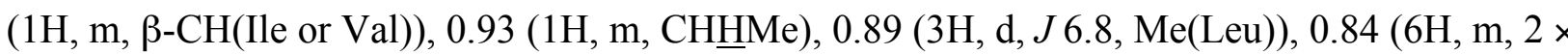
Me(Ile or Val)), 0.73 (3H, d, J 6.8, Me(Leu)), 0.66 (6H, m, $2 \square \mathrm{Me}\left(\right.$ Ile or Val)); $\square_{c}(100 \mathrm{MHz}$; MeOD) 172.3 (C), 172.2 (C), 171.4 (C), 170.5 (C), 156.8 (C), 137.1 (C), 136.9 (C), 130.3 (C), $128.1(\mathrm{CH}), 127.6(\mathrm{CH}), 127.2(\mathrm{CH}), 125.7(\mathrm{C}), 123.8(\mathrm{CH}), 118.3(\mathrm{CH}), 118.0(\mathrm{CH}), 114.5$ $(\mathrm{CH}), 108.5(\mathrm{C}), 66.1\left(\mathrm{CH}_{2}\right), 58.5(\mathrm{CH}), 58.2(\mathrm{CH}), 58.1(\mathrm{CH}), 52.2(\mathrm{CH}), 51.3(\mathrm{Me}), 50.8(\mathrm{CH})$, $37.7(\mathrm{CH}), 31.0(\mathrm{CH}), 27.6(\mathrm{CH}), 27.2\left(\mathrm{CH}_{2}\right), 24.3\left(\mathrm{CH}_{2}\right), 20.8(\mathrm{Me}), 17.7(\mathrm{Me}), 17.5(\mathrm{Me}), 16.7$ (Me), $14.5(\mathrm{Me}), 9.5(\mathrm{Me})$, containing 35\% of an impurity which suggested that epimerization 
had occurred during the reaction. The product was used in the next step without further purification.

\section{(b) D2-17}

An identical procedure to above was followed using D2-16 (30 mg, $0.032 \mathrm{mmol})$. Purification gave D2-17 (12 mg, 56\%) as a colorless solid; $[\square]_{D}^{29} 28.6$ (c 0.21, MeOH); (Found: $\mathrm{MH}^{+}$, 676.3706. $\mathrm{C}_{37} \mathrm{H}_{49} \mathrm{~N}_{5} \mathrm{O}_{7}+\mathrm{H}$ requires 676.3705); $\mathrm{\square}_{\mathrm{H}}(400 \mathrm{MHz}$; MeOD) 7.34 (1H, d, $J$ 8.4, 4-H), $7.26(5 \mathrm{H}, \mathrm{m}, \mathrm{ArH}), 7.04(1 \mathrm{H}, \mathrm{s}, 2-\mathrm{H}), 6.99(1 \mathrm{H}, \mathrm{s}, 7-\mathrm{H}), 6.47(1 \mathrm{H}, \mathrm{d}, J 8.4,5-\mathrm{H}), 4.99(2 \mathrm{H}$, app q, $J$ 10.0, 12.0, $\left.\underline{\mathrm{C}}_{2} \mathrm{Ar}\right), 4.66(1 \mathrm{H}, \mathrm{d}, J$ 4.0, $\square-\mathrm{CH}(\mathrm{Leu})), 4.53(1 \mathrm{H}, \mathrm{d}, J$ 10.0, $\square-\mathrm{CH}(\mathrm{Trp})), 4.43(1 \mathrm{H}$, $\mathrm{d}, J 5.3, \mathrm{\square}-\mathrm{CH}(\mathrm{Val})), 4.23(1 \mathrm{H}, \mathrm{d}, J 11.0, \mathrm{\square}-\mathrm{CH}(\mathrm{Ile})), 3.70(3 \mathrm{H}, \mathrm{s}, \mathrm{OMe}), 3.21(1 \mathrm{H}, \mathrm{m}, \mathrm{-}-$

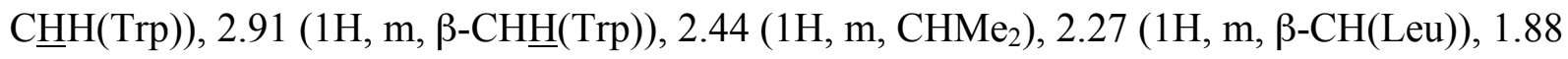

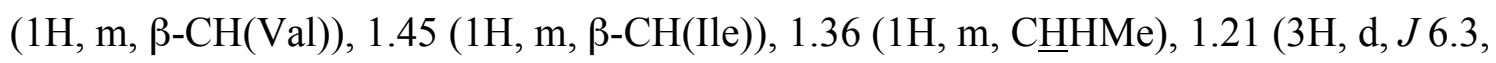
Me(Leu)), 0.95 (1H, m, CHMe), 0.86 (3H, d, J 6.9, Me(Val)), 0.81 (3H, d, J 6.6, CHMe), 0.73 (6H, m, Me(Ile), Me(Val)), 0.51 (3H, d, J 6.3, Me(Leu)); \c (100 MHz; MeOD) 171.6 (C), 171.1 (C), $170.9(\mathrm{C}), 169.9(\mathrm{C}), 156.0(\mathrm{C}), 136.7(\mathrm{C}), 131.5(\mathrm{C}), 128.2(\mathrm{CH}), 128.0(\mathrm{CH}), 127.9(\mathrm{CH})$, $127.5(\mathrm{C}), 126.5(\mathrm{C}), 123.1(\mathrm{CH}), 119.3(\mathrm{CH}), 117.1(\mathrm{CH}), 113.4(\mathrm{CH}), 112.7(\mathrm{C}), 66.9\left(\mathrm{CH}_{2}\right)$, $59.12(\mathrm{CH}), 59.07(\mathrm{CH}), 57.9(\mathrm{CH}), 55.7(\mathrm{CH}), 55.5(\mathrm{CH}), 51.5(\mathrm{Me}), 36.5(\mathrm{CH}), 32.1(\mathrm{CH}), 29.5$ $\left(\mathrm{CH}_{2}\right), 29.1(\mathrm{CH}), 24.6\left(\mathrm{CH}_{2}\right), 21.3(\mathrm{Me}), 20.9(\mathrm{Me}), 17.9(\mathrm{Me}), 17.1(\mathrm{Me}), 14.5(\mathrm{Me}), 9.1(\mathrm{Me})$. 


\section{Stephanotic acid methyl ester 6}<smiles></smiles>

Palladium(II) hydroxide on carbon (20\%; 10mg) was added to a solution of D1-17 (15 mg, 0.022 $\mathrm{mmol})$ in ethanol $(0.5 \mathrm{ml})$. The system was evacuated and flushed with nitrogen 5 times, and evacuated and flushed with hydrogen 5 times. The reaction mixture was left stirring under hydrogen at atmospheric pressure for $2 \mathrm{~h}$, and filtered through Celite. The filter pad was washed with ethanol (3 $\square 10 \mathrm{ml})$ and the combined washings were concentrated. The amine was dissolved in DMF $(0.8 \mathrm{ml})$ and cooled to $0{ }^{\circ} \mathrm{C} .(S)$-Pyroglutamic acid $(4 \mathrm{mg}, 0.031 \mathrm{mmol})$, HOAt (4 mg, $0.029 \mathrm{mmol})$, diethylisopropylamine (10 $\square \mathrm{l}, 0.058 \mathrm{mmol})$ and HATU (11 $\mathrm{mg}, 0.029 \mathrm{mmol})$ were sequentially added to the solution. The reaction mixture was allowed to warm to room temperature overnight and the solvent was removed under vacuum. The residue was dissolved in ethyl acetate $(20 \mathrm{ml})$ and was washed with hydrochloric acid $(1 \mathrm{M} ; 10 \mathrm{ml})$, saturated aqueous sodium hydrogen carbonate $(10 \mathrm{ml})$ and brine $(10 \mathrm{ml})$. The organic layer was dried $\left(\mathrm{MgSO}_{4}\right)$ and concentrated. The residue was purified and the product isolated by normal phase followed by reversed phase preparative HPLC to give stephanotic acid methyl ester 6 ( $2 \mathrm{mg}, 14 \%)$ as a colorless solid; mp 196-201 ${ }^{\circ} \mathrm{C}$, (lit., $\left.{ }^{3} \mathrm{mp} 202-204{ }^{\circ} \mathrm{C}\right) ;[\square]_{D}^{28}-106$ (c 0.09, MeOH), (lit., ${ }^{3}[\square]_{D}^{25}-$ 119 (c 1.7, MeOH)); (Found: $\mathrm{MH}^{+}, 653.3669 . \mathrm{C}_{34} \mathrm{H}_{48} \mathrm{~N}_{6} \mathrm{O}_{7}+\mathrm{H}$ requires 653.3663); $\square_{\mathrm{H}}(400 \mathrm{MHz}$; DMSO) 10.72 (1H, s, NH(Ind)), 8.56 (1H, J, 10.8, NH(Leu)), 8.34 (1H, d, J, 8.5, NH(Ile)), 7.91 (1H, s, NH(PyroGlu)), 7.48 (1H, d, J 8.5, NH(Trp)), 7.41 (1H, d, J 8.5, 4-H(Ind)), 7.03 (3H, m,

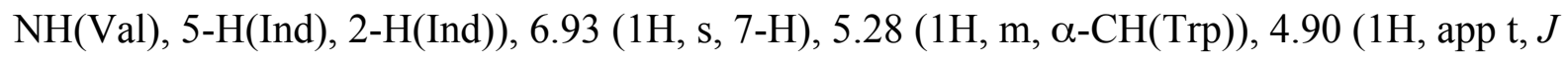


10.8, $\square-\mathrm{CH}(\mathrm{Leu})), 4.16$ (1H, dd, $J$ 9.0, 3.4, \-CH(PyroGlu)), 3.93 (1H, dd, $J$ 7.6, 6.0, ७-CH(Val)), $3.82(1 \mathrm{H}, \mathrm{dd}, J 10.3,8.5, \mathrm{Q}-\mathrm{CH}(\mathrm{Ile})), 3.70(3 \mathrm{H}, \mathrm{s}, \mathrm{OMe}), 3.32(1 \mathrm{H}, \mathrm{m}, \mathrm{-}-$

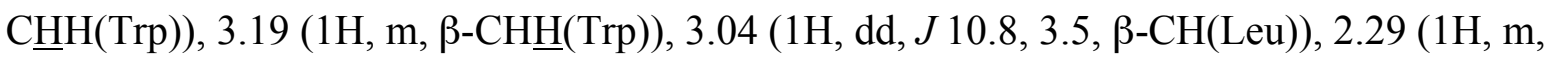

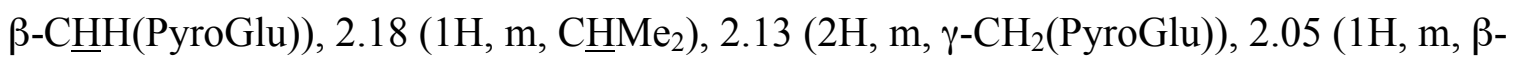

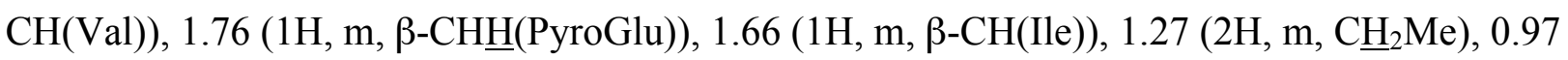
(3H, d, $J$ 6.9, $\mathrm{Me}(\mathrm{Val})), 0.94$ (3H, d, $J$ 6.8, Me(Val)), 0.90 (3H, d, $J$ 7.1, Me(Leu)), 0.82 (3H, d, $J$ 6.8, Me(Leu)), 0.72 (3H, t, $J$ 8.3, $\left.\mathrm{CH}_{2} \mathrm{Me}\right), 0.69$ (3H, d, $J$ 6.9, CHMe); 口c (100 MHz; DMSO) 178.4 (CONH-PyroGlu), 173.2 (CO-PyroGlu), 172.7 (CO-Trp), 171.6 (CO-Val), 170.9 (COLeu), 170.7 (CO-Ile), 137.0 (8-C-Ind), 130.8 (6-C-Ind), 126.5 (9-C-Ind), 124.4 (2-CH-Ind), 120.0

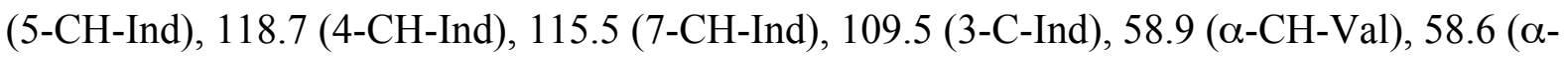

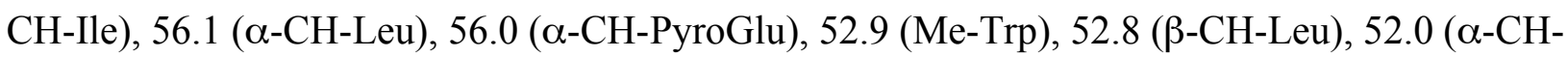

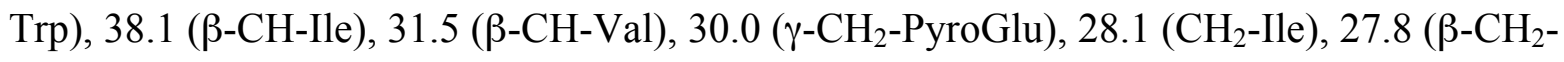

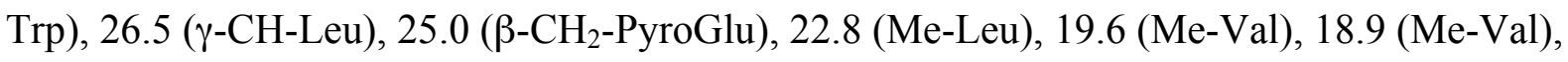
18.3 (Me-Leu), 16.3 (CHMe-Ile), $11.3\left(\mathrm{CH}_{2}\right.$ Me-Ile); $m / z$ (ES) $675\left(\mathrm{M}+\mathrm{Na}^{+}, 38 \%\right), 653\left(\mathrm{M}+\mathrm{H}^{+}\right.$, 26), 360 (100), 338 (87), 204 (31), 150 (47).

Carbon assignments made by HMQC spectroscopy, (quaternary carbons assigned by comparison with the literature report). ${ }^{3}$

\section{Diastereomer of Stephanotic acid methyl ester Diast-6}

An identical procedure to above was followed using D2-17 (12 $\mathrm{mg}, 0.018 \mathrm{mmol})$. Purification of the crude product was by column chromatography ( $2 \%$ up to $5 \%$ methanol / ethyl acetate) to give Diast-6 (4 mg, 34\%) as a colorless solid; mp 220-225 ${ }^{\circ} \mathrm{C} ;[\square]_{D}^{30} 22(c 0.09, \mathrm{MeOH}), \square_{\mathrm{H}}(400$ MHz; MeOD) $7.53(1 \mathrm{H}, \mathrm{d}, J$ 8.4, 4-H), $7.19(1 \mathrm{H}, \mathrm{s}, 7-\mathrm{H}), 7.15(1 \mathrm{H}, \mathrm{s}, 2-\mathrm{H}), 6.64$ (1H, dd, J 8.4, 
1.4, 5-H), $4.96(1 \mathrm{H}, \mathrm{d}, J 3.9, \mathrm{\square}-\mathrm{CH}(\mathrm{Leu})), 4.65(1 \mathrm{H}, \mathrm{d}, J 11.4, \mathrm{\square}-\mathrm{CH}(\operatorname{Trp})), 4.52(1 \mathrm{H}, \mathrm{d}, J 5.3$, ๑-CH(Val)), 4.29 (1H, d, $J$ 11.6, —-CH(Ile)), 4.21 (1H, dd, $J$ 8.4, 6.5, —-CH(PyroGlu)), 3.80 (3H,

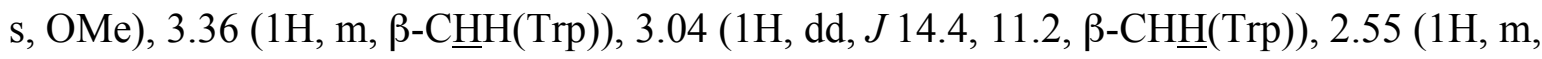
$\left.\mathrm{CHMe}_{2}\right), 2.48(1 \mathrm{H}, \mathrm{m}, \mathrm{\square}-\mathrm{C} \underline{\mathrm{HH}}(\mathrm{PyroGlu})), 2.38$ (3H, m, $\left.\square-\mathrm{CH}(\mathrm{Leu}), \mathrm{CCH}_{2}(\mathrm{PyroGlu})\right), 2.08$ (2H,

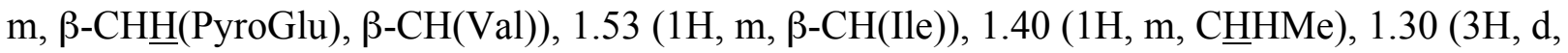
$J$ 6.4, Me(Leu)), 1.01 (3H, d, J 6.8, Me(Val)), 0.96 (1H, m, CHㅐMe), 0.88 (6H, m, Me(Ile), Me(Val)), 0.79 (3H, t, J 7.3, Me(Ile)), 0.63 (3H, d, J 6.6, Me(Leu)); Zc (100 MHz; MeOD) 180.5 (C), 172.8 (C), 171.7 (C), 171.0 (C), 170.1 (C), 169.5 (C), 136.8 (C), 131.5 (C), 126.7 (C), 123.3 $(\mathrm{CH}), 119.8(\mathrm{CH}), 117.4(\mathrm{CH}), 113.2(\mathrm{CH}), 112.6(\mathrm{C}), 59.3(\mathrm{CH}), 58.20(\mathrm{CH}), 58.17(\mathrm{CH}), 57.0$ (CH), $55.2(\mathrm{CH}), 54.4(\mathrm{CH}), 51.4(\mathrm{Me}), 35.9(\mathrm{CH}), 31.6(\mathrm{CH}), 29.4\left(\mathrm{CH}_{2}\right), 29.3\left(\mathrm{CH}_{2}\right), 29.2$ (CH), $25.1\left(\mathrm{CH}_{2}\right), 24.5\left(\mathrm{CH}_{2}\right), 21.2(\mathrm{Me}), 20.8(\mathrm{Me}), 18.0(\mathrm{Me}), 16.8(\mathrm{Me}), 14.4(\mathrm{Me}), 8.8(\mathrm{Me})$.

\section{X-ray crystal data of $( \pm)-12$}

Crystal data

Temperature

Wavelength

Crystal system

Space group

Unit cell dimensions

Volume

Z

Density (calculated)

Absorption coefficient

$\mathrm{F}(000)$
93(2) K

$0.71073 \AA$

Monoclinic

$\mathrm{P} 2(1) / \mathrm{n}$

$\mathrm{a}=14.248(3) \AA \quad \square=90^{\circ}$.

$\mathrm{b}=7.7909(16) \AA \quad \square=108.455(3)^{\circ}$.

$\mathrm{c}=22.151(5) \AA \quad \square=90^{\circ}$.

$2332.5(9) \AA^{3}$

4

$1.243 \mathrm{Mg} / \mathrm{m}^{3}$

$0.087 \mathrm{~mm}^{-1}$

928 
Crystal size

Theta range for data collection

Index ranges

Reflections collected

Independent reflections

Completeness to theta $=25.36^{\circ}$

Absorption correction

Max. and min. transmission

Refinement method

Data / restraints / parameters

Goodness-of-fit on $\mathrm{F}^{2}$

Final $\mathrm{R}$ indices $[\mathrm{I}>2 \operatorname{sigma}(\mathrm{I})]$

$\mathrm{R}$ indices (all data)

Extinction coefficient

Largest diff. peak and hole
$0.2000 \times 0.2000 \times 0.0300 \mathrm{~mm}^{3}$

2.79 to $25.36^{\circ}$.

$-16<=\mathrm{h}<=17,-9<=\mathrm{k}<=8,-26<=\mathrm{l}<=22$

16112

$3944[\mathrm{R}($ int $)=0.0329]$

$92.1 \%$

None

1.0000 and 0.2692

Full-matrix least-squares on $\mathrm{F}^{2}$

3944 / 2 / 299

1.046

$\mathrm{R} 1=0.0368, \mathrm{wR} 2=0.0855$

$\mathrm{R} 1=0.0438, \mathrm{wR} 2=0.0902$

$0.0039(17)$

0.225 and -0.184 e. $\AA^{-3}$ 


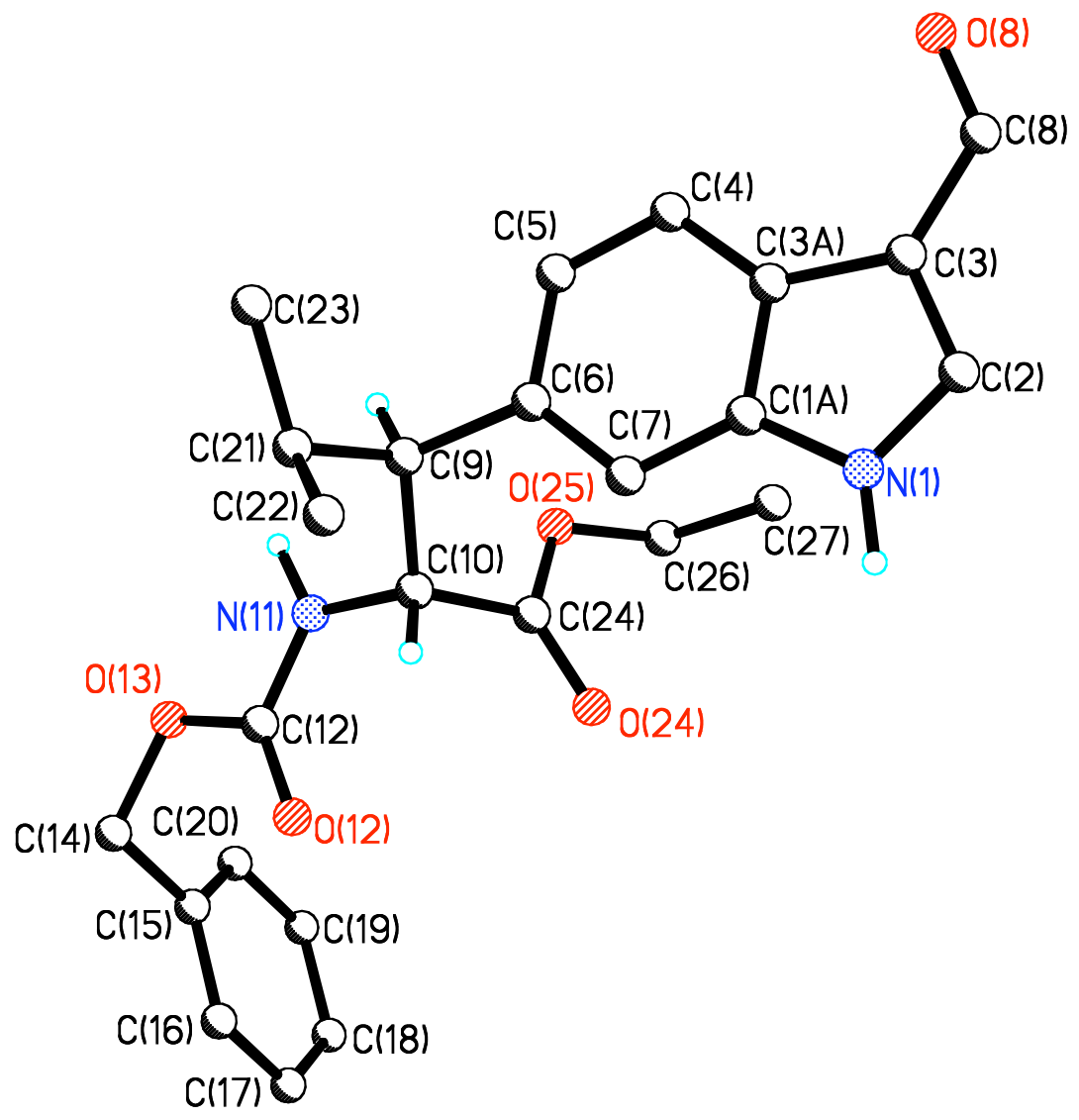




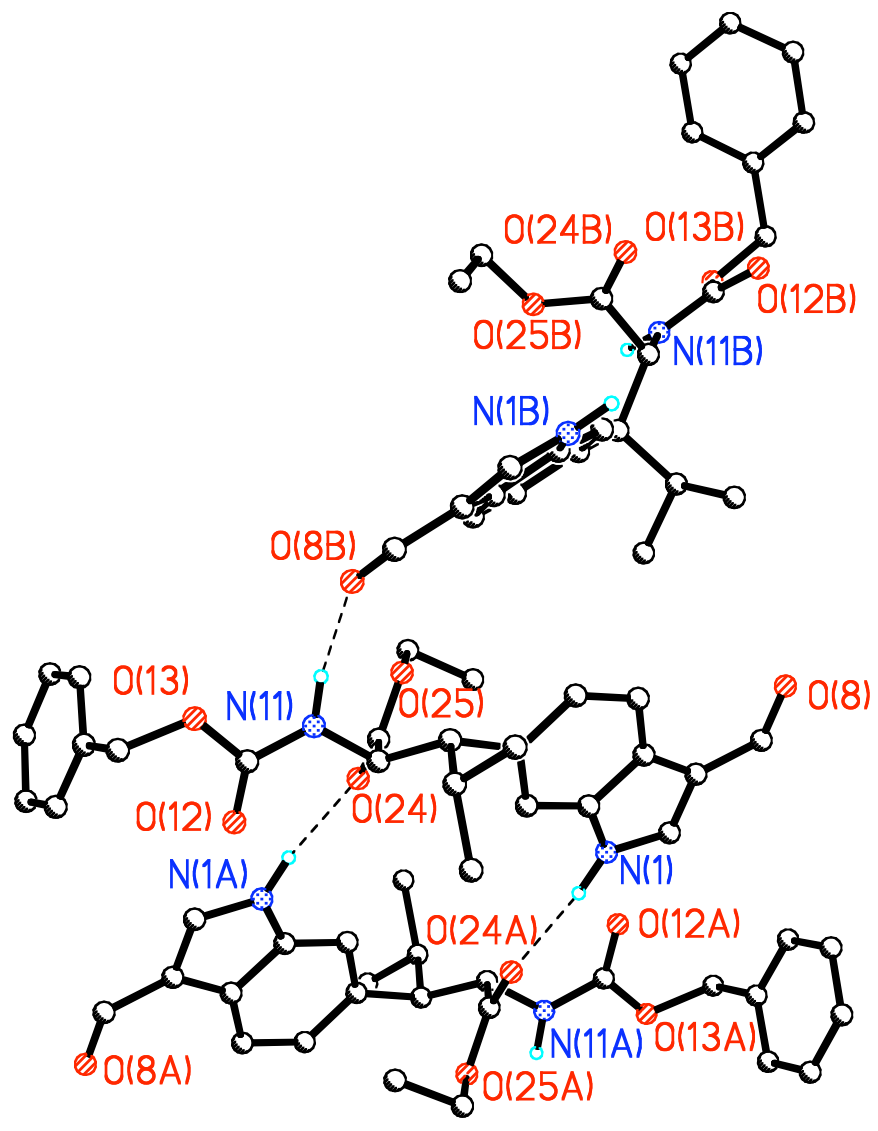

\section{References}

(1) Ikan, R.; Rapaport, E. Tetrahedron 1967, 23, 3823-3827.

(2) Bentley, D. J.; Moody, C. J. Org. Biomol. Chem. 2004, 2, 3545-3547.

(3) Yoshikawa, K.; Tao, S.; Arihara, A. J. Nat. Prod. 2000, 63, 540-542. 